Editorial summary: Interferometric scattering microscopy (iSCAT) enables ultrasensitive label-free imaging and high-speed single particle tracking. This Protocol describes how to construct an iSCAT microscope including total internal reflection single molecule fluorescence capabilities.

Proposed Tweet: \#NewNprot: Label-free single molecule imaging with iSCAT [LINK] \#superres \#imaging \#microscopy

Keywords: interferometric scattering microscopy, iSCAT, label-free imaging, high-speed imaging, TIRF, TIRFM, total internal reflection fluorescence, total internal reflection fluorescence microscopy, single molecule, single molecule imaging, single molecule detection, single particle tracking

\title{
Supporting papers
}

Kukura P, Ewers H, Mueller C, Renn A, Helenius A, Sandoghdar V. High-speed nanoscopic tracking of the position and orientation of a single virus. Nature Methods 2009 6:923-935 doi:10.1038/nmeth.1395

Arroyo JO, Andrecka J, Spillane KM, Billington N, Takagi Y, Sellers JR, Kukura P. Label-Free, All-Optical Detection, Imaging, and Tracking of a Single Protein. Nano Letters 2014 14:2065-2070 DOI: $10.1021 / \mathrm{nl} 500234 \mathrm{t}$

Andrecka J, Ortega-Arroyo JO, Takagi Y, de Wit G, Fineberg A, MacKinnon L, Young G, Sellers JR, Kukura $P$ Structural dynamics of myosin 5 during processive motion revealed by interferometric scattering microscopy. Elife 2015 4:e05413 DOI: 10.7554/eLife.05413\#.dpuf

\section{An Interferometric Scattering Microscope Capable of Simultaneous Total Internal Reflection Fluorescence Imaging}

\author{
Jaime Ortega Arroyo ${ }^{1}$, Daniel Cole ${ }^{1}$, and Philipp Kukura ${ }^{1, *}$ \\ ${ }^{1}$ Physical and Theoretical Chemistry Laboratory, South Parks Road, Oxford OX1 3QZ, UK \\ *email: philipp.kukura@chem.ox.ac.uk, Tel: +44 1865-275401, Fax:+44 1865275410
}

\begin{abstract}
Interferometric scattering microscopy (iSCAT) is a scattering-based imaging modality, which offers a unique combination of imaging speed and precision for tracking nanoscopic labels and enables labelfree optical sensing down to the single-molecule level. In contrast to fluorescence-based imaging, iSCAT does not suffer from limitations associated with dye photochemistry and photophysics or the requirement for fluorescent labeling. Here, we present a protocol for constructing an iSCAT microscope from commercially available optical components and demonstrate its compatibility with simultaneously operational single-molecule, objective-type, total-internal reflection fluorescence microscopy (TIRFM). Given an intermediate level of experience with optics and microscopy, for instance graduate-level familiarity with laser beam steering and optical components, this protocol can be completed in a timeframe of two weeks.
\end{abstract}

\section{Introduction}


The advent of single-molecule optics has enabled the study of nanoscale processes and dynamics previously hidden by the ensemble. Fluorescence detection has been the dominant contrast mechanism for detecting and imaging single molecules over the last 25 years. One of the main advantages of single-molecule fluorescence detection besides its superior background suppression is the wide range of genetically encodable fluorescent proteins. The intrinsic photophysical properties of single emitters such as optical saturation, photobleaching and photoblinking, however, impose fundamental limitations on the achievable photon flux from a single emitter light source ${ }^{1,2}$. As a consequence, high imaging speeds lead to a significant loss in image quality and thus localization precision; in single-particle tracking applications, this translates to a rule of thumb expression relating the localization precision with the imaging speed of $\sim 1 \mathrm{~nm} \mathrm{~Hz}{ }^{1 / 2}$ when significant observation times (>100 ms) are required. Higher imaging speeds are desirable ${ }^{3}$, since motion on the nanoscale at ambient condition usually takes place on much faster, ms - ns timescales. Fluorescence-based imaging and detection also requires labeling of the species of interest, as the majority of molecules are not highly fluorescent and photostable. In addition, fluorescent labels can perturb the intrinsic properties and underlying dynamics of the system under study, for example when investigating micro and nanoscopic phase separation phenomena in lipids, fluorescent dyes have been reported to alter lipid partitioning and distribution.

\section{Development of the protocol}

Owing to these limitations, there have been numerous attempts to move beyond fluorescence as the sole contrast mechanism for single-molecule imaging and detection ${ }^{4-9}$. One approach relies on the detection of scattered light, which is not subject to optical saturation, photobleaching or blinking. Here, the detected photon flux is predominantly a function of the incident power and the scattering cross-section of the object. Gold nanoparticles, which scatter visible light efficiently due to a localized surface plasmon resonance, have been used to replace fluorescent dyes or quantum dots in single-particle tracking applications and provide very high simultaneous spatial and temporal precision. In addition, since any object will scatter light as long as its refractive index differs from the surrounding medium, scattering and extinction detection is capable of non-resonant, label-free imaging and sensing ${ }^{10,11}$. In the extreme case, even a single protein in buffer scatters enough light to enable single-molecule sensing and imaging without the need for any labels ${ }^{12,13}$.

Two main approaches to scattering-based microscopy have emerged over the past decades: purely scattering-based and interferometric-based detection. To illustrate the basic differences between the two, we compare them with respect to a standard epi-fluorescence microscope (Fig. 1a). Detection of pure scattering, commonly referred to as darkfield microscopy, relies on rejecting the illumination light from the detection channel to reveal the object of interest. Darkfield illumination is usually achieved by a mismatch in excitation and detection numerical aperture or by taking advantage of total internal reflection (Fig. 1b). For common-path interferometric-based approaches such as interferometric scattering microscopy (iSCAT) ${ }^{10,14-16}$ where the reference field is produced by a reflection from the glass coverslip due to a change in refractive index at the interface, the background light is collected and recombined together with any scattered light in a reflection geometry enabled by a beamsplitter (Fig. 1c). The choice of a common-path geometry is highly advantageous, as it avoids measurement noise caused by fluctuations in the path lengths travelled by the scattered and reference light fields. The concept of interferometric detection in such a geometry has been explored for decades in interference reflection microscopy ${ }^{17}$ and more recently through reflection interference contrast microscopy. ${ }^{18,19}$ The major difference between those techniques and iSCAT is that the latter uses a coherent light source, which increases the interferometric contrast. 
Regardless of the approach, purely scattering- or interferometric-based, the detected intensity reaching the detector $I_{d}$, can be written as:

$$
\mathrm{I}_{d} \propto\left|\mathbf{E}_{\boldsymbol{r}}+\mathbf{E}_{\boldsymbol{s}}\right|^{2}=\mathbf{E}_{\boldsymbol{i}}^{2}\left[\zeta^{2}+s^{2}+2 \zeta|s| \cos \phi\right],
$$

where $s$ is the complex scattering amplitude, $\varphi$ the phase difference between the scattered and reference fields and $\mathbf{E}_{r}, \mathbf{E}_{s}, \mathbf{E}_{i}$ are the reference, scattered and incident electric fields, respectively. Depending on the detection approach, $\zeta$ denotes either the amplitude of any non-suppressed background for darkfield microscopy or the reflectivity of the interface for iSCAT. The pure scattering term, $\mathrm{s}^{2}$, dominates the detected signal in darkfield microscopy with the remaining terms negligible for sufficiently small scatterers. In iSCAT microscopy, the reference contribution, $\zeta^{2}$, dominates the light intensity at the detector. Furthermore, if the path difference between $\mathbf{E}_{r}$ and $\mathbf{E}_{s}$ is smaller than the coherence length of the illumination source, the electric fields will interfere and the third interferometric term becomes significant. In the limit of a small scatterer, $\mathrm{s}^{2}$ is negligible and the detected intensity in an iSCAT experiment is given by:

$$
\mathrm{I}_{i S C A T} \propto \mathbf{E}_{i}^{2}\left[\zeta^{2}+2 \zeta|s| \cos \phi\right]
$$

The signal of a scattering object in iSCAT therefore manifests itself as a small variation on top of a large background, with a sign that scales with the phase difference between the scattered and reflected fields. In a perfect experiment, shot noise-induced fluctuation of the light intensity at the detector is the sole source of noise in iSCAT images. The associated root-mean-square signal fluctuations scale with the number of detected photons $(N)$ as $1 / V N$, which depend on the incident power and the reflectivity of the sample. In an ideal single-molecule fluorescence measurement, where the shot noise of the detected fluorescence photons determines the imaging noise, the photon flux is limited by the photophysical properties of the emitter. In iSCAT the power of the illumination source limits the photon flux since a constant proportion, determined by $\zeta^{2}$, reaches the detector. This implies that, in principle, arbitrary temporal resolution, localization precision and sensitivity can be achieved by tuning the amount of light incident on the sample for a given exposure time. The scattering cross-section $(\sigma)$ in the limit of objects with diameters, $D<<\lambda$ is given by:

$$
\sigma=s^{2} \propto \varepsilon_{m}(\lambda)^{2} \pi \frac{D^{6}}{4 \lambda^{4}}\left|\frac{\varepsilon_{p}(\lambda)-\varepsilon_{m}(\lambda)}{\varepsilon_{p}(\lambda)+2 \varepsilon_{m}(\lambda)}\right|^{2}
$$

where $\mathrm{D}$ is the particle diameter, $\lambda$ the illumination wavelength and $\varepsilon_{m}$ and $\varepsilon_{p}$ denote the dielectric constant of the medium and particle, respectively. It follows that the dependence of the detected signal on particle diameter is reduced to $D^{3}$ in iSCAT as opposed to $D^{6}$ for darkfield imaging, resulting in a less dramatic drop in scattering signal with a reduction in particle size. Although background light contributions are also present in iSCAT, the reflection from the sample surface is generally much stronger than the spurious reflections and unavoidable background light sources that corrupt image quality in darkfield. Considering the scenario of a shot noise-limited experiment, this distinction between iSCAT and darkfield leads to a difference between the total noise of the system in the presence of these additional contributions. In iSCAT the total noise is determined almost exclusively by the strong reflection at the sample surface and thus remains mostly unaffected by these undesirable contributions; whereas in darkfield these contributions are added in quadrature. Furthermore, these spurious reflections are not part of the sample, are constant and have a welldefined intensity and as a result can be easily accounted for by the process of flat-fielding (see Image Processing section) without compromising image quality. The process of flat-fielding is, in principle, also applicable to darkfield imaging, but the large differences in intensity between background and scattering signals of a nanoparticle and the much stronger size dependence $D^{6}$ vs $D^{3}$, requires detectors with extremely high dynamic ranges to adequately represent the detected intensity of 
each contribution simultaneously, making an efficient subtraction difficult in practice for weak scatterers, although not impossible ${ }^{20}$.

\section{Overview of procedure}

In this protocol, we describe how to construct an iSCAT microscope from commercially available optical components and discuss the critical experimental details required to perform iSCAT with the highest levels of sensitivity and imaging speed. We focus on sample illumination by beam scanning as we have found it to be the most versatile and flexible method, although a number of other approaches have been reported ${ }^{16,21}$. In addition, we show how to reduce experimental complexity and make the technique easily compatible with state-of-the-art single molecule fluorescence imaging using objective-type total internal reflection illumination ${ }^{22}$.

\section{Advantages}

The key features that make iSCAT an attractive tool are:

- Indefinite observation times, given that the technique relies on the detection of scattered light and thus does not suffer from the adverse effects of optical saturation, photoblinking or photobleaching as encountered in fluorescence.

- Temporal resolution and localization precision are decoupled from one another, because under optimal performance the signal to noise ratio of a scatterer in iSCAT is shot noise-limited, and as a result can be increased arbitrarily by collecting more photons.

- High sensitivity to weak scatterers compared to darkfield microscopy, $D^{3}$ vs. $D^{6}$ size dependence.

- Intrinsically label-free technique, as any nano-object with a different refractive index than the surrounding will scatterer light and is therefore detectable.

- Compatible with single-molecule fluorescence detection.

\section{Limitations}

The potential of iSCAT is vast because the limits of sensitivity, speed and localization precision are determined by the availability of appropriate camera sensors and design of the sample area instead of the technique itself. From an implementation perspective, we found that control over sample stability represents the main system limitation. Nevertheless, possibly the greatest advantage of iSCAT over fluorescence, i.e. its label-free imaging ability, is also its largest limitation. The lack of specificity to differentiate nano-objects beyond the intensity of the signal and the characteristic dynamic behavior of the object under study poses a considerable experimental challenge when dealing with complex, multi-component systems.

This limitation can be partly alleviated with correlative fluorescence imaging or differential imaging in the presence of a substrate with specificity for the target molecule. Therefore, it is necessary to consider the type of sample and the experimental conditions. Ideally the nano-object of interest should have a discernible signal, or have the property of containing both an active and inactive state under which differential imaging is operational, all while having a sample with a welldefined background signal that can be extracted and removed. In principle, it is feasible to study samples with highly heterogeneous scattering backgrounds if such samples have: a time invariant or very slowly varying background, the signal of interest moves on a time scale much faster than the background and the coherence length of the illumination source is sufficiently large for the interference term to occur.

\section{Applications}

For a carefully designed experimental setup as described here, iSCAT is capable of shot noise-limited detection of small scattering objects down to single protein molecules without the 
need for fluorescent labelling ${ }^{13}$. We have recently taken advantage of the unique capabilities of iSCAT to perform single-particle tracking assays with simultaneous high temporal resolution and nmprecision across multiple biophysical systems. For instance, iSCAT can be applied to the field of molecular motors as we have shown with the direct observation of myosin 5 moving along actin ${ }^{23}$ (Fig. 2a) where we tracked $20 \mathrm{~nm}$ gold labels with simultaneous $\mathrm{nm}$ spatial and $\mathrm{ms}$ temporal precision. Another field with ample applications for iSCAT is membrane biophysics where we have shown that the imaging speed can be improved towards $50 \mathrm{kHz}$ while maintaining $\mathrm{nm}$ precision ${ }^{24}$ (Fig. 2b) or even approaching frames rates close to $1 \mathrm{MHz}$ with an appropriate camera ${ }^{25}$.

Focusing on sensitivity rather than speed, we have demonstrated that iSCAT can detect and image single proteins without labels and with $\mathrm{nm}$ precision ${ }^{12}$, such as shown here for myosin 5 (Fig. 2c). This achievement paves the way for label-free bio-sensing applications ${ }^{13}$. Moreover, given that iSCAT is an intrinsically label-free imaging platform it can be used to study nanoscale phase transitions with high sensitivity, such as the formation of supported lipid bilayers on glass substrates $^{26}$ (Fig. 2d).

\section{Experimental design}

Setup considerations for an optimal iSCAT microscope A complete iSCAT experimental setup is presented in Figure 3. For iSCAT illumination, we prefer standard diode lasers as they possess much shorter coherence lengths than diode-pumped solid state (DPSS) or HeNe lasers. Although the last two types of lasers provide much cleaner beam profiles, the long coherence length leads to interference of almost any reflections within the experimental setup resulting in additional imaging noise ${ }^{15}$. The short coherence length of diode lasers limits these interferences to the sample region and to some of the objective optics. To improve the image quality, we spatially filter the diode output by focusing it through a pinhole or by coupling it into a single mode fiber followed by collimation to a beam diameter of $1 \mathrm{~mm}$. The beam then passes through two acousto-optic deflectors (AODs, Gooch \& Housego), which rapidly scan the beam in two dimensions. Two planoconvex lenses (TL1 and TL2) arranged in a $4 f$ geometry image the beam deflections generated by the AODs into the back focal plane (BFP) of a high numerical aperture microscope objective (Olympus PlanApo, 100x, 1.4 NA). We mount the diode laser to ensure a P-polarized output after the AODs and pass it through a polarizing beamsplitter (PBS), which separates the illumination and detection channels. A quarter wave plate (QWP) circularly polarizes the illumination beam before being directed into the back aperture of the microscope objective by $M 1$ and $M 2$, which enable precise coupling into the microscope. A final mirror at 45 degrees to the plane of the table, termed the objective-coupling mirror (OCM), directs the illumination light into the objective. The objective collects the light reflected at the glass/water interface together with the back-scattered light from the sample, which becomes S-polarized after a second pass through the QWP. The PBS then reflects the scattered and reflected light into the detection arm of the setup where they are focused onto a CMOS camera with a lens (L1). An adjustable mirror (M7) ensures that the light travels parallel to the table to avoid the introduction of optical aberrations upon passing through the imaging lens. Mirror pairs $M 1 / 2, M 3 / 4$ and $M 5 / 6$ are used to ensure reproducibility and ease of alignment.

Beam scanning vs wide-field illumination The choice of scanning a focused beam across a sample to effectively achieve wide-field illumination is a priori surprising as this could in principle be achieved by simply focusing the collimated laser output into the back focal plane of the objective as in standard wide-field fluorescence microscopy. However, this latter approach generates significant interference fringes and contributions from speckle noise, which deteriorate the iSCAT image quality $^{15}$. The main source of these back-reflections, are multiple optical elements present in a high NA objective. Both speckle noise and interference fringes would not pose a problem if they remained constant during a typical exposure time as they could be removed by image processing, 
but that would require an interferometrically stable setup. Achieving interferometric stability on a microscope while possible, drastically increases the experimental complexity of the setup. Although wide-field illumination would in principle be desirable, we have not found it feasible in practice beyond fields of view on the order of $10 \times 10 \mu \mathrm{m}^{2}{ }^{13,15}$ Instead, scanning a weakly focused beam across the sample, avoids these fringes and results in what appears to be an evenly illuminated sample region to the imaging (CMOS) camera, which operates with an exposure time much longer than the scanning period of the AODs (12.5 $\mu \mathrm{s})$. Repeated scanning during a single exposure also reduces the effect of laser intensity noise in a fashion analogous to lock-in detection. We reset the beam deflections after each image is acquired by synchronizing the AOD operation with the camera exposure, to ensure that the beam undergoes the same scanning pattern after each camera exposure and therefore maintain a constant illumination profile.

When adjusting the beam size entering the microscope, which can be achieved by choosing appropriate collimation optics after mode cleaning, we recommend a final spot size at the sample on the order of 1-2 $\mu \mathrm{m}$, which provides an ideal compromise between speckle reduction and low peak illumination intensity. The final beam size required will vary with the magnification and thus size of the entrance aperture of the microscope objective. Beam-scanning decreases the effect of speckle on the quality of the image, however, the non-interfering background still remains, which results in an offset intensity that can significantly lower the contrast of the scatterer and thus lower the signal to noise ratio of a measurement. Although a detailed characterization of the effect of the noninterfering background is currently not attainable, the contrast range targets for $20 \mathrm{~nm}$ gold particles indicative of a well-aligned setup are $4.5 \%$ at $660 \mathrm{~nm}$ and $9.0 \%$ at $445 \mathrm{~nm}$.

Combination with single molecule TIRF One of the great advantages of iSCAT is its compatibility with fluorescence imaging and its ease of implementation on existing custom-built or commercially available epi-fluorescence or TIRF microscopes. Adding a single dichroic mirror (DM2) in the beam path of the iSCAT setup converts an iSCAT microscope into a dual TIRF-iSCAT setup (Fig. 3). The only requirement is that the dichroic mirror separates the iSCAT wavelength from both fluorescence excitation and emission. A single optic thereby completely divides the experimental setup into an iSCAT and a TIRF part, which can be operated simultaneously or individually. In other words, construction of the iSCAT setup described here yields a highly efficient TIRF microscope at the low expense of a few optics, a light source and a sensitive camera. In principle, multiple combinations of TIRF and iSCAT wavelengths are possible, and can be selected based on the desired application. Here, we illustrate combined TIRF and iSCAT microscopy using blue fluorescence excitation and red iSCAT illumination as we have found it to enable single-molecule TIRF imaging of common fluorophores such as ATTO 488, Alexa 488, GFP and quantum dots without suffering perturbations from the iSCAT channel. One specific example for the performance of simultaneous iSCAT and TIRF is shown in Figure 4, where a single myosin $V$ molecule has been doubly labeled with a $20 \mathrm{~nm}$ gold nanoparticle and a quantum dot, respectively. ${ }^{23}$

Choosing a long pass dichroic mirror (DM2, $638 \mathrm{~nm}$ cutoff wavelength, Thorlabs cat. no. DMLP638) efficiently separates a $660 \mathrm{~nm}$ iSCAT channel and a $400-620 \mathrm{~nm}$ fluorescence channel. A second dichroic mirror (DM1, $490 \mathrm{~nm}$ cutoff wavelength, Thorlabs, cat no. DMLP490) further separates the TIRF excitation $(462 \mathrm{~nm})$ from the emitted fluorescence providing a $500-620 \mathrm{~nm}$ detection window. We generally prefer dichroic mirrors fabricated on thick optical substrates to minimize interference patterns often generated from two reflections of a thin substrate. In addition to standard fluorescence filters, a long pass (F1, Semrock $635 \mathrm{~nm}$ EdgeBasic $^{\top \mathrm{M}}$, cat. no. BLP01-635R25) and bandpass filter (F2, Omega 560BP80 RAPIDBAND, cat. no. RPB520-600) minimize residual background.

In this protocol we consider the scenario of a fluorescence excitation laser that is coupled out from a single mode fiber with a $4 \times$ objective placed approximately $500 \mathrm{~mm}$ away from the back 
focal plane of the microscope objective. Focusing into the back focal plane for wide-field illumination involves moving the collimation objective away from the fiber output. In this way, we optimize the achievable field of view and homogeneity of the excitation field on the sample while ensuring excellent reproducibility of the beam path. Mirrors DM1 and DM2 direct the fluorescence excitation beam into the objective, and lateral displacement of DM1, which is mounted on a linear translation stage, enables total internal reflection. The objective collects the fluorescence emission, which is then imaged onto an EMCCD with a lens of appropriate focal length (L2) to achieve the desired magnification.

Focus stabilization Using a fiber-coupled light source with an excellent spatial mode and drift-free alignment is particularly beneficial because it enables straightforward implementation of stabilizing the sample-to-objective distance, which is important for high-sensitivity iSCAT imaging. Our approach operates under the principle that axial displacements between the sample and the objective translate into lateral beam displacements of a totally internal reflected beam, which is imaged onto a camera ${ }^{27}$. In practice we achieve this with a pick-off mirror (D) before DM1 and a cylindrical lens (CyL) to image the beam onto a CMOS camera as a line focus. For the feedback to operate ideally, it is important to minimize beam pointing instabilities and air currents as these cause unwanted beam displacements that could be mistaken for focus drift or vibration. The position of the line focus is calibrated against sample-objective displacement and an active feedback loop maintains the focus position. Using this approach, we routinely achieve $<10 \mathrm{~nm}$ stabilization of the objective-sample distance.

Choice of camera and magnification We found that CMOS cameras best suit iSCAT applications as they offer the highest achievable frame rates, versatility in the selection of the region of interest, large full-well capacities and generally lower cost than their CCD counterparts. It is useful to characterize the response of the camera so that every measurement is performed in the shot noiselimited regime, since the specifications of the CMOS camera will inherently determine the range of possible applications. We usually characterize the sensor properties such as the read-out noise, the shot noise-limited regime, the camera digital units to photo-electron conversion and the full-well by calculating the photon transfer curves ${ }^{28}$.

With respect to the shot noise-limited performance of the camera, the number of photoelectrons each pixel can detect before saturation (full well capacity) defines the choice of magnification for the microscope and as a result the maximum achievable sensitivity per single image. For most applications we recommend pixel-to-pixel signal variations of $0.1 \%$ with an effective pixel size on the order of $60 \mathrm{~nm}$, which requires detecting $10^{6}$ photoelectrons per effective pixel. Unfortunately, most CMOS sensors lack such a high dynamic range and instead possess full-well capacities in the tens to hundreds of thousands of photoelectrons. This limitation can be circumvented by increasing the nominal magnification and subsequently binning the counts from adjacent pixels.

To illustrate this concept, consider the parameters of our most frequently used camera (Photonfocus MV-D1024-160-CL-8, 1024×1024 pixels, $10 \mu \mathrm{m}$ pixel size, $0.2 \mathrm{Mē}$ full well capacity). For this camera, collection on the order of $1 \mathrm{Me} /$ pixel requires $3 \times 3$ binning $(3 \times 3 \times 0.2 \mathrm{Me}=1.8 \mathrm{Me})$. To achieve an effective pixel size of $60 \mathrm{~nm}$ after software binning thus requires a 500x magnification, resulting in $20 \mathrm{~nm} /$ pixel. Such magnification values are conveniently achieved using a $1 \mathrm{~m}$ focal length imaging lens together with a 100x magnification objective. The attainable field of view at this magnification is $20 \times 20 \mu \mathrm{m}^{2}$, which we find sufficient for most applications. Importantly, software pixel-binning relaxes the need for bit depth beyond 10-bits thereby ensuring fast readout. This level of sensitivity suffices for most single-particle tracking measurements, but can easily be improved further by temporal frame binning. The camera discussed above achieves a full chip frame rate of 
100 frames per second at 4 ms exposure, meaning that the effective well-depth increases to 18 $\mathrm{Me} / \mathrm{pixel}$ upon time-averaging to a bandwidth of $10 \mathrm{frames} / \mathrm{s}$. This corresponds to shot noiseinduced fluctuations on the order of $2.4 \times 10^{-4}$, which is an order of magnitude smaller than the iSCAT signal corresponding to a $500 \mathrm{kDa}$ protein ${ }^{12,13}$.

Data acquisition We employ LabVIEW to interface the cameras, synchronize the AODs, and manipulate the sample stage in three dimensions, but any suitable software platform can be used. The cameras should operate continuously and preferably under a ring buffer mode for the highest image quality and system performance since it enables real-time image processing. We strongly recommend real-time image processing, specifically flat-fielding (see Image Processing section), for efficient day-to-day operation of any iSCAT microscope. Furthermore, special consideration should be taken when running at frame rates beyond the deterministic time-scale of the interfacing hardware to avoid frame-skipping. For most desktop personal computers this time-scale is on the order of milliseconds and for field-programmable gate arrays (FPGA) platforms on the order of tens of microseconds. Many solutions that operate at higher frames rates exist, but are entirely specific to the choice of camera, frame-grabber, and computer system; and are thus beyond the scope of this protocol. We have found that the combination of frame grabbers and LabVIEW operating in ring buffer mode allows us to acquire images at up to $50 \mathrm{kHz}$ continuously and in real time.

Microscope hardware The microscope is ideally assembled on an optical table with active stabilization to minimize the coupling of low frequency noise from the environment into the microscope system. In addition, we found it imperative that the objective is fixed to a heavy stage. This dampens vibrations and improves stability, as the objective is the single most important and sensitive optic in the setup. We usually implement this by drilling a hole into an aluminum optical breadboard, held by four large pedestals, and tapping it complementary to the thread found in the objective (usually the Royal Microscopical Society, RMS, thread). The second most important aspect involves the sample mount and respective translation stage systems. There are many available options that vary from manual translation stages fitted with piezo actuators to commercially available nano-positioning systems with strain gauges. Irrespective of the choice, as long as the sample stage mount satisfies the following two criteria they can be used in any iSCAT microscope: Firstly, axial positioning of the sample should be fine-tunable to within a few nanometres by active feedback for the most sensitive measurements. Secondly, the stages should not introduce or should be able to correct for sample tilt so that lateral motion of the sample does not change the focus position. In addition, we recommend enclosing the entire setup into compartments to avoid unwanted noise in the form of vibrations or air currents due to fan elements or temperature gradient with the additional benefit of decreasing background light for single-molecule fluorescence detection. Each AOD also requires a radio frequency module that relies on a function generator. The function generator serves two purposes: to determine the amplitude, offset, shape and frequency of the beam scanning and to synchronize the camera and the AODs.

Image processing The raw images from an iSCAT microscope contain static features that are intrinsic to the microscope and yet extrinsic to the sample (Fig. 5a). The inhomogeneous baseline of this image results from non-uniform illumination, non-uniform pixel response, fixed pattern noise, spurious back-reflections, unwanted interference between back-reflections or imperfections of optical elements. Features of this nature are time-invariant and do not change as a function of lateral sample displacement, which means they can be removed. We call the process of isolating and subsequently removing these signatures through the normalization of raw images by a temporal median image: flat-fielding ${ }^{29}$. We acquire the temporal median image by simultaneously translating the sample laterally while recording a stack of about 100 images under two specific conditions. First, the focus position of the sample must remain constant as some of the background signatures are focus-dependent. Secondly, the speed of the lateral displacement must be sufficiently high so that 
on average each pixel does not contain information from a specific sample feature (e.g. $10 \mu \mathrm{m} / \mathrm{s}$ at $100 \mathrm{~Hz}$ ). In this way, computing the median for each pixel across the stack of acquired images generates a representative background lacking any sample specific features as illustrated for a single line of the image in Figure 5b. Given that the median background is generated from a stack of 100 individual images, shot noise-induced background fluctuations are significantly reduced compared to a single image. Division of the raw by the median image not only improves the image contrast by removing all stationary background features, but also does not introduce any additional noise into the image, resulting in a drastic drop in baseline inhomogeneity (Fig. 5c). It is important to note that the choice of a division (normalization) over subtraction is performed so that the signal contrast in the images is not affected by variations in pixel intensities due to intrinsic static features produced by the sample.

Flat-fielding can only correct for truly static background features that do not belong to the sample, because the median image is acquired over several seconds whilst moving the sample area. Under realistic experimental conditions, other time-varying signal fluctuations such as unsynchronized beam-scanning, laser mode hops, sample focus drift and large out-of-focus scattering signals can lead to inhomogeneous baselines, which fluctuate on the tens of millisecond to second timescale (Fig. 4d). These features extend over areas much larger than a typical pointspread function, and can therefore be extracted by an additional image processing tool, which we denote as pseudo flat-fielding. The pseudo flat-field image is obtained by setting the kernel size significantly larger than the typical point-spread function and with the operation to calculate either the median or mean. The resulting image only contains the features that are much larger than the diffraction-limit. Much like the flat-field case, division of the raw image by the pseudo flat-field image improves the image quality considerably and produces an image with an almost homogeneous, shot-noise limited baseline (Fig. 5d).

Regardless of the above image processing routines, intrinsic sample features such as surface roughness contribute to a constant and static scattering background that limits the achievable signal to noise ratio required for the detection of very weak scatterers. This scattering background can overwhelm the signal of interest, especially when the contrast of the scattering object is very small. As before, this background is constant and can be removed by two distinct yet complementary approaches termed: dynamic imaging (Fig. 5e) and differential imaging (Fig. 5f). In dynamic imaging, scatterers of interest that are mobile are treated as foreground elements and isolated. In the simplest and computationally least demanding scenario, when the scatterers move sufficiently far so that on average they spend less than half of the time on the same pixels, static background elements can be extracted and removed by a temporal median approach. For a sequence of images acquired when the sample position is held constant, a background image containing only static features intrinsic to the sample can be generated by calculating the median value of each pixel across the image stack (Fig. 5e). Subtraction of this median from each image in the acquired stack, yields an image dominated by shot noise and containing signals from dynamic features in the sample. For extremely weak scatterers, shot noise dominates the observable signal, but the SNR of the dynamic features can be improved by pixel binning and temporal averaging as discussed in the section: Choice of camera and magnification. The effectiveness of dynamic imaging is closely related to the speed at which the object moves and the time resolution of the measurement. To illustrate this, let us consider the 2D Brownian diffusion of a small label imaged at a frame rate of $1000 \mathrm{fps}$ for an observation time of $1 \mathrm{~s}$. To efficiently remove all static features and reveal mobile scatterers with temporal median-based dynamic imaging, the object must move a distance of at least three times the radius of a diffraction limited spot (DLS) away from its starting point in $500 \mathrm{~ms}$. For high NA objectives the full width at half maximum of a DLS is on the order of $200 \mathrm{~nm}$, therefore we require the PSF of the object to have moved $(0.6 \mu \mathrm{m})^{2}=0.36 \mu \mathrm{m}^{2}$ in $500 \mathrm{~ms}$. Using the relation MSD $=4 \mathrm{Dt}$ 
we can conclude that the diffusion coefficient of the object must be on the order of $0.18 \mu \mathrm{m}^{2} / \mathrm{s}$ or above for dynamic imaging to perform optimally.

Dynamic imaging is prone to fail when the signals of interest are immobile, for example when detecting very small particles such as single proteins in a bio-sensing assay. If the immobile features possess the characteristic akin to an active and inactive state, such as the binding or unbinding of an analyte, we can use differential imaging to generate shot noise-limited images with the signal of interest ${ }^{13,26}$. Differential imaging consists of subtracting one image from a second, temporally offset one. Figure $5 f$ shows two iSCAT images, one recorded at time $t$ and the other at a fixed time interval, $\Delta t$, later. Subtraction of the former image from the latter removes all stationary features and reveals any dynamics in the sample occurring within the specified time interval.

Sample drift and time-varying signals from background scatterers limit both approaches. However, as long as these processes take place on a slower time-scale than the rate at which the scattering background is generated, they can be considered inconsequential. Ultimately, the image processing routines described in this section perform the critical task of transforming the raw data into shot noise-limited images containing diffraction-limited spots that closely resemble the data acquired under a state-of-the-art single-molecule fluorescence microscope. As a result, decades of research and development of algorithms for spot detection, super-resolution and trajectory linking can be directly transferred and applied to iSCAT.

\section{Materials}

CRITICAL. All optical components, such as lens mounts, pedestals, clamping forks, posts, postholders and base plates can be purchased from Thorlabs, although any suitable manufacturer of optomechanical components can be employed.

CRITICAL: It is highly encouraged that the assembly of the sample stage be performed in advance to avoid delays in the construction of the iSCAT microscope.

\section{Laser sources}

- iSCAT illumination: $658 \mathrm{~nm}$ diode laser (Lasertack, cat. no. LDM-658-300)

- TIRF illumination and autofocus: 462 nm diode laser (Lasertack, cat. no. LDM-462-1400-C)

\section{Microscope assembly and sample stage}

- Optical table with active stabilization (Thorlabs, Nexus Optical Table with Sealed Holes, $1 \mathrm{~m}$ $\times 2 \mathrm{~m} \times 310 \mathrm{~mm}$, with $600 \mathrm{~mm}$ Tall Active Isolator Legs, cat. no. T1020CK). Although optional, we highly recommend the use of an optical table with active stabilization because it significantly reduces the coupling of low frequency vibrations from the surroundings into the microscope setup.

- Stainless steel 1.5 inch posts, $125 \mathrm{~mm}$ height, base adapters and clamping forks for mounting breadboard to optical table (Thorlabs, cat no. P125/M) (x4)

- Aluminum optical breadboard with RMS threaded hole to mount detection objective. (Thorlabs, cat no. MB2020/M)

Critical: Thread the RMS according to the specifications of the objective. Perform this in advance and ensure that the objective has no significant tilt while mounted to the breadboard.

- $x y$-translation stage for course adjustment of lateral sample position (OptoSigma, cat no. $122-0245,120 \mathrm{~mm} \times 120 \mathrm{~mm}$ ) 
- Linear translation stage with piezo, for axial adjustment and focus stabilization. (Thorlabs, cat. no. NFL5DP20/M) (or any commercial $x y z$ piezo stage combined with a microscopic translation system can be used, e.g. Mad City labs)

- 90 degree $L$ mounting bracket (Thorlabs, cat no. AP90/M)

- Post-holder base (Thorlabs, cat. No. BA2)

- High precision kinematic mirror mounts (LIOP-TECH cat no. SR-100-100R-2-BU)

- Aluminum sample holding plate (Fig. 7b).

Critical: Prepare this sample holder in advance according to the specifications of the objective, linear translation stage and the glass coverslips.

\section{iSCAT Channel}

- Mounted aspheric lens for focusing diode laser into single mode fiber. (Thorlabs cat. No. A110TM-A)

- Z-axis translation mount for cage system (Thorlabs, cat. No. SM1Z)

- Externally SM1-Threaded terminated fiber adapter (Thorlabs, cat No. SM1FC) (x2)

- SM1-Threaded post mountable cage plate (Thorlabs, cat. No. CP02/M) (x2)

- Cage assembly rods (Thorlabs, cage system and construction series)

- Polarization maintaining single mode fiber, $620-850 \mathrm{~nm}, 2 \mathrm{~m}$ (Thorlabs, cat. No. P1-630PMFC-2)

- Linear translation stage to mount fiber-coupling objective (Opto-Sigma, cat. No. TADC-651)

- High precision kinematic mirror mounts (LIOP-TECH cat no. SR-100-100R-2-BU) (x10)

- Broadband dielectric mirrors $400-700 \mathrm{~nm}$ (Thorlabs, cat no. BB1-EO2) (x10)

- Acousto optic deflectors for beam scanning (AOD, Gooch\&Housego cat. no AODF 4120-3) $(x 2)$

- Variable frequency RF driver for the AODs (Gooch\&Housego cat. no AODR 1110FM-3) (x2)

CRITICAL: Ensure that all accessory electronic components to the AODs are available and functional according to the manufacturer's specifications. This should be performed in advance.

- Rotation platform to mount AOD (Thorlabs, cat. no. RP01/M)

- N-BK7 plano-convex lenses for telecentric relay system (N-BK7 Plano-Convex Lenses, AR Coating: $350-700 \mathrm{~nm}, \mathrm{f}=400 \mathrm{~mm}$, Thorlabs, cat no. LA1172-A) (x2)

- Broadband polarizing beamsplitter cube, $420-680 \mathrm{~nm}$, for separation of illumination and detection paths (PBS, Thorlabs, cat no. PBS251)

- Mounted achromatic quarter-wave plate, $400-800 \mathrm{~nm}$, for circular polarization of illumination beam (QWP, Thorlabs, cat no. AQWP05M-600)]

- Mounted achromatic half-wave plate, $400-800 \mathrm{~nm}$, for maximizing AOD diffraction efficiency (Thorlabs, cat. no. AHWP05M-600)

- Continuous Rotation Mount for $\varnothing 1 / 2 "$ Optics to house quarter-wave plate (Thorlabs, cat. no. $\mathrm{RSP05/M)}$

- Continuous Rotation Mount for $\varnothing 1 "$ Optics to house half-wave plate (Thorlabs, cat. no. RSP1/M)

- $45^{\circ}$ Mount for $\varnothing 1^{\prime \prime}$ optics to house objective coupling mirror (OCM, Thorlabs cat no. H45)

- Long pass filter (F1, Semrock 635 nm EdgeBasic ${ }^{T M}$, cat. no. BLP01-635R-25)

- Visible achromatic doublet lens for imaging detection beam onto CMOS camera, $f=1000$ $\mathrm{mm}$ (L1, Thorlabs, cat no. AC508-1000-A,). The choice of focal length can be adjusted to select the desired magnification.

- Post mounted iris for alignment (Thorlabs, cat. no. ID12/M) (x4)

- Shearing interferometer (Thorlabs, cat. no. SI100)

\section{TIRF/Autofocussing Channel}


- Mounted aspheric lens for focusing diode laser into single mode fiber. (Thorlabs cat. No. A110TM-A)

- Z-axis translation mount for cage system (Thorlabs, cat. No. SM1Z)

- Externally SM1-Threaded terminated fiber adapter (Thorlabs, cat No. SM1FC) (x2)

- SM1-Threaded post mountable cage plate (Thorlabs, cat. No. CP02/M) (x2)

- Cage assembly rods (Thorlabs, cage system and construction series)

- Single mode fiber patch cable, 405-532 nm, $2 \mathrm{~m}$ (Thorlabs, cat. No P1-405B-FC-2)

- Linear translation stage to mount fiber-coupling objective (Opto-Sigma, cat. No. TADC-651)

- High precision kinematic mirror mounts (LIOP-TECH cat no. SR-100-100R-2-BU) (x4)

- Broadband dielectric mirror $400-700 \mathrm{~nm}$ (Thorlabs, cat no. BB1-EO2) (x2)

- Dichroic mirror to separate iSCAT and TIRF illumination wavelengths, $638 \mathrm{~nm}$ cutoff wavelength, (DM2, Thorlabs cat. no. DMLP638)

- Linear translation stage for achieving total internal reflection (Opto-Sigma, cat. No. TADC651)

- Dichroic mirror to separate fluorescence excitation and emission paths, $505 \mathrm{~nm}$ cutoff. (DM1 longpass, Thorlabs cat. no. DMLP505)

- Bandpass filter (F2, Omega 560BP80 RAPIDBAND, cat. no. RPB520-600)

- Imaging lens, visible achromatic doublet, focal length dependent on EMCCD camera (Thorlabs, Unmounted Achromatic Doublets, AR Coated: $400-700 \mathrm{~nm}$ )

- Pick-off mirror for autofocusing channel (D, Thorlabs, cat. no. BBD1-EO2)

- Cylindrical lens (CyL, $f=400 \mathrm{~mm}$, Thorlabs, cat.no. LJ1363RM-A)

\section{Objectives}

- Detection - Olympus PlanApo, 100x, 1.4 NA

- Fiber outcoupling, iSCAT - Olympus Plan Achromat, 20x, 0.4 NA (Thorlabs, cat. no. RMS20X)

- Fiber outcoupling, TIRF - Olympus Plan Achromat, 4x, 0.10 NA (Thorlabs, cat. no. RMS4X)

\section{Detection Cameras}

- CMOS camera for iSCAT detection (CMOS1, Photonfocus MV-D1024-160-CL-8)

- EMCCD camera for fluorescence detection (Andor iXon3 860 EMCCD)

- CMOS camera to perform autofocusing (CMOS2.Thorlabs, cat no. DCC1545M)

\section{Hardware}

- Rigol DG1000 Wavefunction Generators (x2)

- NI Framegrabber, exact specification will depend on camera and software employed (for a dual channel system we use: NI PCle-1430).

\section{Software}

- National Instruments, LabVIEW 2011 onwards with the Vision Development and Vision Acquisition modules, or any suitable software platform.

\section{REAGENTS}

- Milli-Q water.

- Ethanol (Sigma, cat. no. 02860-1L)

- Isopropanol (Sigma, cat. no. 190764-1L)

- PBS tablets (Sigma, cat. no. P4417-50TAB)

- Biotin BSA (Sigma, cat. no. A8549-10MG)

- $\quad$ BSA (Sigma, cat. no. A2153-10G)

- Streptavidin coated AuNPs (20 nm, $40 \mathrm{~nm}$ ) (Innovo biosciences, InnovaCoat ${ }^{\circledR}$ GOLD - $20 \mathrm{~nm}$ and $40 \mathrm{~nm}$, cat. nos. 251-1000 and 250-1000) 
- Fluorescent beads TransFluoSpheres ${ }^{\circledR}$ Carboxylate-Modified Microspheres, $0.1 \mu \mathrm{m}, 488 / 560$ (Life Technologies, cat.no. T-8872)

- Borosilicate glass coverslips (No.1.5, 24 × 50 mm, VWR) and (No. 1.5, 24 × 24 mm, VWR)

\section{REAGENT SETUP}

1xPBS solution: PBS is $10 \mathrm{mM}$ phosphate buffer, $2.7 \mathrm{mM}$ potassium chloride, $137 \mathrm{mM}$ sodium chloride. Mix 1 PBS tablet in 0.2 I of milliQ $\mathrm{H}_{2} \mathrm{O}, \mathrm{pH} 7.4$. Filter the solution and store it at RT for up to 6 months.

Biotin-BSA solution $(0.1 \mathrm{mg} / \mathrm{ml})$ : Add $0.5 \mathrm{mg}$ of biotin-BSA into $50 \mathrm{ml}$ of $1 \times$ PBS buffer solution. Filter the solution and store $4^{\circ} \mathrm{C}$ for up to 2 years.

BSA blocking solution $(10 \mathrm{mg} / \mathrm{ml})$ : Add $50 \mathrm{mg}$ of biotin-BSA into $5 \mathrm{ml}$ of $1 \times$ PBS buffer solution. Filter the solution and store $4^{\circ} \mathrm{C}$ for up to 2 years.

\section{Procedure}

\section{Alignment of iSCAT illumination path TIMING - 3 days}

1. Decide on the position of the microscope head assembly and optical components on the optical table according to Figure 3, allowing for easy access and ample space for the alignment of illumination and detection paths for the iSCAT and TIRF channels. $1 \mathrm{~m}^{2}$ of optical table space is sufficient.

2. Install the red diode laser (Lasertack, cat. no. LDM-658-300) for iSCAT illumination on the optical table. Ensure the laser has a proper heat sink and is secured to the table to avoid unwanted thermal currents and beam pointing instabilities.

CAUTION: Class IV lasers can cause serious eye injury, wear appropriate eye protection and ensure that only trained personal are allowed to operate the laser.

3. Assemble the fiber coupling according to Figure 6 .

4. Spatially filter the output of the diode laser by coupling it into a polarization-maintaining single mode fiber with two adjustable mirrors. The input polarization must be matched to the slow axis of the fiber to ensure a high polarization extinction ratio. The orientation of the slow axis of the fiber is indicated by the narrow key on the fiber end, rotate the fiber so as to match this to the laser polarization.

5. Couple out of the fiber with a $20 x$ objective mounted on a linear translation stage to give a high quality Gaussian beam profile. Ensure the beam is collimated from the fiber using a shear plate. Ensure the beam exits the objective P polarized by rotating the end of the fiber such that the intensity of the beam transmitted through a polarizing beam splitter (PBS) is maximized.

6. Adjust the size of the beam using a telescopic lens system. Select the focal lengths of each lens such that the resulting beam diameter is approximately $1 \mathrm{~mm}$ at full width half maximum. Ensure the beam is not displaced by the telescope using an iris and that the resulting beam is collimated using a shear plate. 
7. Install two broadband dielectric mirrors in high precision kinematic mounts as shown in Figure 3. Direct the beam along the mounting holes in the optical table and in a plane parallel to the table using two irises. We suggest a height of $75 \mathrm{~mm}$ for all beam paths in the microscope.

8. Install two broadband mirrors, (M3 and $M 4$ ), in high precision kinematic mounts allowing ample space to install the AODs in between. Direct the beam in a straight line parallel to the table as shown in Figure 3.

CRITICAL STEP: it is important that the beam deflections caused by the AODs are correctly mapped into the BFP of the objective by the 4-f telecentric lens system. Therefore, allow for ample space to place the remaining optical components so that the AODs can be positioned approximately $4 f$ away from the BFP of the objective. In this protocol we consider the scenario where the telecentric lens system is composed of two $f=400 \mathrm{~mm}$ lens. In this case the AODs are placed $1600 \mathrm{~mm}$ from the BFP of the objective.

9. Insert another two adjustable mirrors (M5 and M6) and direct the beam straight and in a plane parallel to the table as shown in Figure 3.

10. Position the broadband polarizing beam splitter (PBS) after M6 such that the illumination beam is transmitted through the face opposite to the indicated entrance face. This ensures that the returning image beam from the sample is reflected by the dielectric beamsplitting coating applied to the hypotenuse of one of the two prisms that make up the cube rather than passing through the cement used to bind the two prisms.

CAUTION: The PBS will cause multiple reflections; ensure that these are blocked to avoid spurious beams entering the eyes and wear appropriate eye protection.

11. Insert DM2 (638 $\mathrm{nm}$, long pass) into the beam path at $45^{\circ}$ and ensure the beam passes through the less reflective side (confirm either by manufacturer label or by observing the back reflection pattern). If only the iSCAT capability is desired, this step can be skipped.

CAUTION: The DM2 optic will cause multiple reflections, ensure that these are blocked to avoid spurious beams entering the eyes and wear appropriate eye protection.

12. The beamsplitter and DM2 will displace the beam. It is critical that the beam travels straight and parallel to the plane of the table after these two optics. Correct for the beam deflections caused by the PBS and DM2 using M5 and M6 and two irises.

13. Direct the beam vertically upwards and perpendicular to the table with a reflective mirror (OCM, Thorlabs, cat no. BB1-EO2) in a high stability kinematic mount set at 45 degrees to the plane of the table (Thorlabs cat no. $\mathrm{H} 45$ ).

CAUTION: Sending the laser beam away from the plane of the table considerably increases the risk of eye injury by accidental viewing. Ensure appropriate eye protection is worn.

14. Install the quarter wave plate (QWP) in a rotation mount (Thorlabs, cat. no. RSP05) and introduce into the beam path after the OCM to circularly polarize the illumination beam.

CAUTION: Placing the QWP in any different position will decrease the detection efficiency. 
15. Construct the microscope head assembly, as shown in Figure 7 , by threading an optical breadboard ( $200 \times 200 \mathrm{~mm}$, Thorlabs cat. no. MB2020/M)) complementary to the detection objective thread (RMS). Attach the breadboard to four pedestals (1.5 inch, $125 \mathrm{~mm}$, Thorlabs, cat. no. P125/M) as shown in Figure 7a. Secure the $x y$ OptoSigma translation stage such that the aperture is centered over the objective thread. Then mount the $z$ translation stage using an L-bracket and a base plate on top of the Opto-Sigma $x y$ stage (Figure 7b). Secure the microscope assembly to the optical table such that the objective thread is centered above the OCM.

16. Screw a high stability kinematic mirror mount with two M4 threads tapped in the front face into the $z$ stage as shown in Figure $7 \mathrm{~b}$. Next secure the sample mount onto the mirror holder. This mirror mount can be used to adjust the tilt in the sample plane.

CRITICAL STEP: the objective is the most important optic in the setup and must be securely mounted to a heavy stage to avoid the coupling of unwanted vibrations into the microscope. Ensure that all components in the microscope assembly are securely fastened to the breadboard.

17. Place a flat reflective mirror on top of the objective thread. This will generate a reflection that should approximately overlap with the incident illumination beam. Adjust the OCM such that this back reflection is overlapped with the incident beam. This ensures that the vertical beam path is straight. Remove the mirror and place a target on the ceiling at the position projected by the beam after this adjustment.

CRITICAL STEP: The alignment of the illumination beam is critical to the final image quality. Therefore to ensure proper alignment it is imperative that the beam travels straight through the objective along its optical axis.

18. Insert the detection objective into the vertical beam path by screwing it into the breadboard and positioning the microscope assembly above the OCM. The beam should be focused tightly by the objective.

CAUTION: The beam can emerge from the objective at an angle if the beam is not correctly aligned, and can cause eye injury.

19. Place a $\mathrm{f}=400 \mathrm{~mm}$ plano-convex lens into the beam approximately one focal length $(400$ $\mathrm{mm}$ ) away from the objective to focus the beam into the back focal plane of the objective. The beam should now appear as a spot on the ceiling.

20. Translate the lens back and forth along the optical axis of the beam to find the point at which the beam spot on the ceiling has the smallest diameter and then secure the lens at this position. At this point, the lens is deemed to be conjugate to the BFP of the objective and thus correctly focused into the objective.

21. Remove the objective and identify the laser spot on the ceiling. Reposition the lens perpendicular to the beam path so that the beam hits the target previously placed there (step 17). 
22. Reinsert the objective and move the microscope assembly such that the beam projected by the objective hits the ceiling target. Then, secure the microscope assembly in this position with pedestals onto the optical table.

\section{Alignment of iSCAT detection path TIMING- 1 day}

23. Remove TL2 from the illumination path such that a collimated beam underfills the back aperture of the microscope objective. Mark the position of the lens before removing it as it will be placed back later.

24. Place a drop of immersion oil onto the objective and a blank glass coverslip on the sample mount. Translating the sample in $z$ should produce a focused spot reflected by the PBS, corresponding to the back reflection from the glass air interface. Rotate the quarter wave plate to maximize the intensity of the reflected beam. TROUBLESHOOTING

25. Using an adjustable mirror placed in a high stability kinematic mount (M7), steer the reflection from the polarizing beam splitter and direct the beam straight and parallel to the plane of the table.

26. Place the camera (Photonfocus MV-D1024-160-CL-8) such that the beam hits the center of the sensor chip and the plane of the sensor is perpendicular to the incident beam.

27. Observe the resulting beam profile on the camera. The microscope is deemed correctly aligned when the profile of the beam focuses concentrically, with radial symmetry and without lateral displacements as the sample is brought in and out of focus (Supplementary Videos 1 and 2). If this is not the case, reiterate steps 17-22. TROUBLESHOOTING

28. Introduce the imaging lens (L1) such that the lens is conjugate with the plane of the camera sensor chip, i.e. $f=1000 \mathrm{~mm}$. It is important that the introduction of this lens does not displace the position of the beam on the camera so as to avoid the introduction of aberrations such as coma and astigmatism. TROUBLESHOOTING

29. Place the long pass filter (F1) before the camera perpendicular to the incident beam.

30. Introduce two target irises into the illumination beam path after M5 and M6, such that the beam passes through the center of both. These will act as a reference to regain alignment following a change in beam pointing in the illumination channel.

\section{Alignment AODs scanning illumination TIMING - 2 days}

31. Reintroduce TL2 into the illumination path (at the position marked in step 23) ensuring not to displace then beam.

32. Install TL1 ( $f=400 \mathrm{~mm}$ ) $800 \mathrm{~mm}$ away from TL2 as shown in Figure 3. Ensure that the introduction of this lens does not displace the beam and that the beam is collimated after TL2. 
33. Install the two acousto-optic deflectors (AOD) according to the manufacturer's instructions and connect the function generators to the modulation input of the radio frequency modules.

34. Place one AOD in the beam path approximately one focal length $(400 \mathrm{~mm})$ away from TL1 such that the beam is travelling through the center of the crystal after the two adjustable mirrors (Figure 3).

CRITICAL STEP: the two AODs must be positioned such that the center between them is conjugate with the back focal plane of TL1.

35. Apply a constant voltage offset from the function generator to the radio frequency module to achieve $50 \%$ of the deflection maximum. The AOD will deflect the incoming beam into many diffraction orders.

36. Adjust the angle of the crystal element with respect to the incident beam so as to maximize the output of the first diffraction order to about $70 \%$ of the incident power. At the Bragg angle, the intensity of all other diffraction orders will be zero. Adjust the crystal angle so as to minimize the contributions from the higher diffraction orders.

CRITICAL STEP: Note that the AODs only efficiently diffract if the incident polarization is parallel to the acoustic wave propagation and the output polarization is rotated by $90^{\circ}$, so introduction of a half-wave plate before the first $A O D$ may be required to achieve optimal transmission efficiency.

37. Secure the second AOD on a rotation platform perpendicular to the first as shown in Figure 8. Adjust the angle of the crystal element by turning the rotation mount so as to maximize the output of the first diffraction order as in step 36.

38. Use the two high precision kinematic mirror mounts placed immediately after the second AOD (M3 and M4) to direct the deflected beam in a straight line in a plane parallel to the table using two irises through TL1 and TL2.

39. Using M5 and M6, direct the beam through the target irises set in step 30 in order to realign the illumination beam straight through the objective.

40. Confirm the realignment by imaging a blank glass coverslip on the camera. The beam should go in and out of focus with radial symmetry as in step 27.

41. In order to scan the beam across the sample and achieve a homogenous illumination profile in the detector plane, the focused beam must be scanned many times over the sample during a single camera exposure. To achieve this, externally trigger the function generators from the frame grabber/camera to only scan during the camera exposure and ensure that the two function generators are phase locked. This is denoted as 'Burst mode' on the function generators. For most applications scanning the two AODs in a sawtooth fashion at 83 and $79 \mathrm{kHz}$ (Figure 9) should suffice for imaging speeds up to $1 \mathrm{kHz}$. The number of scan cycles per exposure time should be set according to the equation $\mathrm{Ns}=$ Scanning frequency $(\mathrm{Hz}) \times$ Frame time (s). For example, given a $4 \mathrm{~ms}$ exposure time the AOD channel operating at $83 \mathrm{KHz}$ should undergo $\mathrm{Ns}=(83000 \mathrm{~Hz}) \times(0.004 \mathrm{~s})=332 \mathrm{cycles}$. Both the absolute and relative frequencies should be chosen to induce the smallest detectable fluctuations in the 
background light intensity on the time-scale of the camera exposure time. The scanning amplitude will depend on the desired region of interest on the camera.

TROUBLESHOOTING

\section{Alignment of TIRF Channel TIMING - 2 days}

CRITICAL: If the TIRF imaging option is not desired steps 50-53 can be omitted. Furthermore DM1 in step 45 can be replaced with a mirror. The remaining steps are used for the axial stabilization.

42. Install the $462 \mathrm{~nm}$ laser diode for TIRF illumination on the optical table, ensuring that it has a proper heatsink and is secured.

CAUTION: Class IV lasers can cause serious eye injury, wear appropriate eye protection and ensure that only trained personnel are allowed to operate the laser.

43. Couple the TIRF illumination into a single mode fiber to clean the mode.

44. Couple out of the fiber using a $4 x$ objective mounted on a linear translation stage to produce a Gaussian beam profile. Ensure the beam from the objective is collimated using a shearing interferometer.

45. Install DM1 in a high stability kinematic mirror mount and secure on a linear translation stage as shown in Figure 3. The direction of travel of the translation stage should allow lateral displacement of the beam.

46. Overlap the iSCAT and TIRF illumination paths with the adjustable mirrors DM1 and DM2.

47. Translate the $4 \times$ objective such that the TIRF excitation beam focuses into the back focal plane of the objective to achieve epi-illumination. The beam is deemed to be focused correctly into the BFP when the diameter of the beam emerging from the objective is the smallest when observed on the ceiling.

48. There should be a faint transmission through DM2 corresponding to the back reflection of the epi-fluorescence excitation beam. This can be used to align the fluorescence emission onto the EM-CCD. Ensure that this beam is travelling in a plane parallel to the table using two adjustable mirrors before introducing the imaging lens ( $L 2$, visible achromatic doublet, Thorlabs, focal length can be selected to achieve desired magnification).

49. With the choice of focal length in mind to obtain a magnification equivalent to $50-90 \mathrm{~nm}$ per EM-CCD camera pixel, center the EM-CCD onto the alignment beam and place the imaging lens to focus the beam onto the camera.

CRITICAL STEP: The fluorescence emission must travel through (L2) straight along the optical axis otherwise optical aberrations are introduced such as coma and astigmatism. TROUBLESHOOTING

50. Assess the quality of the PSF by introducing an appropriate emission filter (F2) and imaging a sample containing a sparse density of surface tethered fluorescent beads. TROUBLESHOOTING 
51. When the sample is in focus, displace the fluorescence excitation beam with the translation stage on which DM2 is mounted until total internal reflection occurs, which can be observed by an intense beam returning from the sample after DM2. This should result in a reduction in background noise due to a reduction in contributions from out of focus fluorescence and an increase in the fluorescence intensity. Fine-tune the displacement of DM2 until the cleanest reflected beam profile is obtained.

\section{Alignment Of Axial Stabilization Channel TIMING - 1 day}

52. Pick off the totally internal reflected beam with a $D$ shaped pick-off mirror placed after DM2 and direct this beam towards a second CMOS camera.

53. Focus this beam onto the CMOS camera with a cylindrical lens. Adjust the position of the cylindrical lens along the beam path such that the width of the line focus on the camera is approximately 20 pixels wide.

54. Use M9 to adjust the angle of the beam incident to the cylindrical lens. Aim to enter the lens at a slightly oblique angle with respect to the plane of the table and the optical axis of the lens. In this way, lateral movements in the reflected beam are more effectively translated into lateral movement in the line focus on the camera chip.

55. Translate the sample along the optical axis, the line focus should move laterally across the camera chip.

\section{Sample Preparation \\ TIMING - 1 day}

CRITICAL: The sample described in this section corresponds to a minimal assay that is used for the sole purpose of assessing the performance of the microscope and the image processing routines. Preparation of other assays may require different optimization procedures.

56. Clean a glass coverslip (No. 1.5, $24 \times 50 \mathrm{~mm}$ ) by successive rinsing with milliQ water, ethanol, isopropanol and again with milliQ water. Dry under a stream of nitrogen.

57. Using double sided sticky tape, securely fix a second, smaller glass coverslip (No. $1.5,24 \mathrm{x}$ $24 \mathrm{~mm}$ ) on top of the cleaned coverslip to create a channel.

58. Place a drop of immersion oil on the objective and insert the coverslip into the sample holder.

CRITICAL STEP: We assume from now on that the user constantly checks the detector output.

59. Flow PBS buffer into the chamber and verify the glass surface is clean. TROUBLESHOOTING

60. Rinse the chamber with a $0.1 \mathrm{mg} / \mathrm{ml}$ solution of biotin-BSA solution and incubate for 5 minutes. TROUBLESHOOTING

61. Wash the flow channel with PBS buffer and then introduce $10 \mathrm{mg} / \mathrm{ml} \mathrm{BSA}$ blocking solution to passivate the remaining glass surface. Incubate for 5 minutes. TROUBLESHOOTING 
62. Wash the flow channel with PBS buffer. TROUBLESHOOTING

63. Add streptavidin functionalized gold nanoparticles, which should bind specifically to the biotinylated BSA.

TROUBLESHOOTING

64. Bring the sample into focus and acquire a median flat field image. Apply flat field correction to the image. This should yield an image that looks similar to that shown in Figure 10a. TROUBLESHOOTING

\section{Troubleshooting}

Troubleshooting advice is provided in Table 1.

Table 1: Troubleshooting

\begin{tabular}{lllll}
\hline Step & Problem & Possible reason & Solution \\
\hline 27 & $\begin{array}{l}\text { Astigmatic beam profile } \\
\text { confocal illumination. }\end{array}$ & $\begin{array}{l}\text { Incorrect alignment through objective/tilt in } \\
\text { sample or objective. }\end{array}$ & $\begin{array}{l}\text { Ensure the beam travels straight } \\
\text { through objective. }\end{array}$ \\
$\begin{array}{l}28,49, \\
50\end{array}$ & $\begin{array}{l}\text { Point spread function } \\
\text { astigmatic profile }\end{array}$ & displays & $\begin{array}{l}\text { Misalignment of the objective or imaging } \\
\text { lens. }\end{array}$ & $\begin{array}{l}\text { Ensure the beam travels straight } \\
\text { through objective and the imaging } \\
\text { lens. }\end{array}$
\end{tabular}

Faulty or a very low quality-imaging filter. Swap filters.

50 Point spread function departs significantly from a Gaussian profile.

41 Intensity fluctuations and beating patterns in the image.
Presence of air bubbles in the sample or in the immersion oil.

Laser experiences significant mode hopping.

AODs are not synchronized

Dirty optics

Large static objects in the ima
are independent of the sample

Large contrast objects that move

together with the sample

Positional fluctuations in the image.

Air currents / thermal gradients present in the setup.

Loose optomechanics.

Displacement of an interface near the surface of the glass.

Back reflections interfering with each other.
Gently press coverslip or move to a different sample area.

Couple laser into a fiber.

Check the external trigger and the output signal from the function generators with an oscilloscope.

Clean all optics thoroughly

Repeat step 56

Enclose setup and divide into compartments to isolate thermal gradients.

Ensure stage and objective are tightly secured

Increase the volume of solution in the sample

Reduce beam diameter and/or 
increase focal length.

Misalign the system slightly by rotating the telecentric lens TL2 offaxis from the normal plane of the incident beam.

Gold particles do not bind to the surface

Density of gold particle binding is too high

Poor real-time flat field correction.

Complete loss of focus between coverslips and autofocus issues.
Issues with surface coverage of the biotinylated BSA

Non-specific binding of gold particles

Insufficient blocking of the surface with BSA or too high coverage of the biotin-BSA surface.

Insufficient focus control during the collection of a temporal median filter.

Sample has not been displaced sufficiently for the temporal median filter to isolate the extrinsic features.

Presence of bubbles in the sample or immersion oil.

Significant change in the weight or flex of coverslip.
Check for beam or camera chip clipping.

Increase the concentration of biotinylated BSA.

Check expiry date of the gold particles

Decrease biotin-BSA incubation time or concentration.

Check the sample stage for cross-talk. components to laterally move the sample during the acquisition of the flat field.

Clean the objective and re-apply the immersion oil.

Ensure that no other objects have been placed on top of the coverslip during acquisition.

Check whether equal pressure is applied to hold the coverslip in place.

Adjust the position of the imaging lens along the optical axis (preferentially the one with the shortest focal length)
Different focus positions be
iSCAT and fluorescence channels

Localization precision curve as a function of illumination intensities does not follow a shot noise limited
Imaging lens from one of the two channels was not placed at the correct distance away from the camera sensor.

Presence of drift, particles not fully immobilized or instability of the sample stage.
Check real-time feedback control of the sample stage position. 


\section{TIMING}

Construction and alignment of a combined iSCAT-TIRF microscope is straightforward and can be completed by a graduate student with prior experience in laser beam steering and optical components in approximately 2 weeks, although this time scales with the experience of those building the microscope, and the time taken for fabrication of parts for the sample stage and microscope assembly.

Steps $1-21$, coupling of laser diode into fiber and alignment of iSCAT illumination path: 3 days Steps $21-28$, alignment of iSCAT detection path: 1 day

Steps $29-39$, alignment AOD scanning illumination: 2 days

Steps $40-49$, alignment of TIRF channel: 2 days

Steps $50-53$, alignment of autofocusing channel: 1 day

Steps $54-62$, sample preparation and image acquisition: 1 day

\section{Anticipated results}

This protocol provides the details for the assembly and implementation of a combined interferometric scattering and single-molecule total-internal-reflection fluorescence microscope. By following this protocol carefully it is possible to image small scattering labels with shot noise limited localization precision and high temporal resolution simultaneously. Figure 10a shows the results anticipated when imaging $20 \mathrm{~nm}$ gold nanoparticles immobilized on a glass substrate and successfully applying the flat field correction in real time with $445 \mathrm{~nm}$ illumination. In addition to immobilized particles, variations in the background representative of the roughness of the coverslip surface should be visible and serve as a quick sensitivity test of the microscope. At an illumination wavelength of $658 \mathrm{~nm}$ the particle contrast for $20 \mathrm{~nm}$ gold should be on the order of $4 \%$. To assess the shot noise-limited behavior of the setup, two adjacent immobilized particles are localized and their positions plotted as a function of time (Fig. 10b, upper panel). Drift contributions are removed by comparing the fluctuations in the distance between the two nanoparticles (Fig. 10b, lower panel). The nominal localization precision is determined from the spread in the distribution of the distance fluctuations (Fig. 10c, inset). By measuring the localization precision at different illumination intensities (or exposure times) the shot noise-limited dependence can be evaluated and serve as a reference for future measurements (Fig. 10c).

The fluorescence microscope capabilities should be comparable to those detailed in a recent protocol $^{30}$ and we recommend using such as a reference for optimizing the fluorescence part of the iSCAT-TIRF microscope. The results highlight the main features of this setup, however higher temporal resolution can be achieved by adding an unscanned iSCAT channel ${ }^{24,25}$. Furthermore the iSCAT and fluorescence channels can be registered to perform colocalization experiments ${ }^{10}$.

This protocol has primarily focused on the application of iSCAT to the field of biophysics. Looking forward and considering the high sensitivity in detecting small changes in scattered light, iSCAT will likely become an ideal tool for investigating nanoscopic structural dynamics featuring small variations in refractive index. ${ }^{31}$ Future studies in this direction will benefit extensively from the integration of tools and concepts from plasmonics and microfluidics and transform iSCAT into a benchmark approach that complements existing ensemble techniques. 
From a technological perspective, the current boundaries are set by the available camera sensor technology and the limitations arising from coherent imaging artefacts intrinsic to laser illumination: speckle noise and unwanted interference patterns. Improvements in temporal resolution and sensitivity will follow from the development of cameras with higher full well capacities and higher read-out rates. Similarly, the image quality and sensitivity will extensively benefit from the arrival of commercially available options of random laser illumination technology. ${ }^{32}$

\section{Author Contributions Statements}

J.O.A and D.C built the optical system and performed the experiments. P.K. supervised and conceived the protocol. J.O.A, D.C and P.K. wrote the manuscript.

\section{Acknowledgements}

J.O.A was supported by a scholarship from CONACyT (scholar: 213546) and PK by an ERC starting grant (NanoScope). The data underpinning this report is freely available at http://ora.ox.ac.uk.

\section{Competing financial interests}

The authors declare no competing financial interests.

\section{References}

1. Moerner, W. E. \& Fromm, D. P. Methods of single-molecule fluorescence spectroscopy and microscopy. Rev. Sci. Instrum. 74, 3597 (2003).

2. Schmidt, T., Schuetz, G. J., Baumgartner, W., Gruber, H. J. \& Schindler, H. Characterization of Photophysics and Mobility of Single Molecules in a Fluid Lipid Membrane. J. Phys. Chem. 99, 17662-17668 (1995).

3. Saxton, M. J. \& Jacobson, K. Single-particle tracking: applications to membrane dynamics. Annu. Rev. Biophys. Biomol. Struct. 26, 373-399 (1997).

4. Boyer, D. Photothermal Imaging of Nanometer-Sized Metal Particles Among Scatterers. Science 297, 1160-1163 (2002).

5. Berciaud, S., Cognet, L., Blab, G. A. \& Lounis, B. Photothermal heterodyne imaging of individual nonfluorescent nanoclusters and nanocrystals. Phys. Rev. Lett. 93, 257402 (2004).

6. van Dijk, M. A., Lippitz, M. \& Orrit, M. Far-Field Optical Microscopy of Single Metal Nanoparticles. Acc. Chem. Res. 38, 594-601 (2005).

7. van Dijk, M. A. et al. Absorption and scattering microscopy of single metal nanoparticles. Phys. Chem. Chem. Phys. 8, 3486 (2006).

8. Ignatovich, F. \& Novotny, L. Real-Time and Background-Free Detection of Nanoscale Particles. Phys. Rev. Lett. 96, 013901 (2006).

9. Hong, X. et al. Background-Free Detection of Single $5 \mathrm{~nm}$ Nanoparticles through Interferometric Cross-Polarization Microscopy. Nano Lett. 11, 541-547 (2011).

10. Kukura, P. et al. High-speed nanoscopic tracking of the position and orientation of a single virus. Nat. Methods 6, 923-927 (2009).

11. Ewers, H. et al. Label-Free Optical Detection and Tracking of Single Virions Bound to Their Receptors in Supported Membrane Bilayers. Nano Lett. 7, 2263-2266 (2007).

12. Ortega Arroyo, J. et al. Label-free, all-optical detection, imaging, and tracking of a single protein. Nano Lett. 14, 2065-2070 (2014).

13. Piliarik, M. \& Sandoghdar, V. Direct optical sensing of single unlabelled proteins and superresolution imaging of their binding sites. Nat. Commun. 5, 4495 (2014).

14. Lindfors, K., Kalkbrenner, T., Stoller, P. \& Sandoghdar, V. Detection and Spectroscopy of Gold Nanoparticles Using Supercontinuum White Light Confocal Microscopy. Phys. Rev. Lett. 93, 
037401 (2004).

15. Jacobsen, V., Stoller, P., Brunner, C., Vogel, V. \& Sandoghdar, V. Interferometric optical detection and tracking of very small gold nanoparticles at a water-glass interface. Opt Express 14, 405-414 (2006).

16. Ortega Arroyo, J. \& Kukura, P. Interferometric scattering microscopy (iSCAT): new frontiers in ultrafast and ultrasensitive optical microscopy. Phys. Chem. Chem. Phys. 14, 15625-15636 (2012).

17. Curtis, A. The mechanism of adhesion of cells to glass A study by interference reflection microscopy. J. Cell. Biol. 20, 199-215 (1964).

18. Ploem, J. S. Reflection-contrast microscopy as a tool for investigation of the attachment of living cells to a glass surface. Mononuclear phagocytes in immunity, infection and pathology 405-421 (1975).

19. Contreras-Naranjo, J. C., Silas, J. A. \& Ugaz, V. M. Reflection interference contrast microscopy of arbitrary convex surfaces. Appl. Opt. 49, 3701-3712 (2010).

20. Weigel, A., Sebesta, A. \& Kukura, P. Dark Field Microspectroscopy with Single Molecule Fluorescence Sensitivity. ACS Photonics 1, 848-856 (2014).

21. Kukura, P., Celebrano, M., Renn, A. \& Sandoghdar, V. Imaging a single quantum dot when it is dark. Nano Lett. 9, 926-929 (2008).

22. Axelrod, D. in Methods in cell biology 89, 169-221 (Elsevier, 2008).

23. Andrecka, J. et al. Structural dynamics of myosin 5 during processive motion revealed by interferometric scattering microscopy. elife 4, e05413 (2015).

24. Spillane, K. M. et al. High-speed single-particle tracking of GM1 in model membranes reveals anomalous diffusion due to interleaflet coupling and molecular pinning. Nano Lett. 14, 5390 5397 (2014).

25. Lin, Y.-H., Chang, W.-L. \& Hsieh, C.-L. Shot-noise limited localization of single $20 \mathrm{~nm}$ gold particles with nanometer spatial precision within microseconds. Opt Express 22, 9159 (2014).

26. Andrecka, J., Spillane, K. M., Ortega Arroyo, J. \& Kukura, P. Direct observation and control of supported lipid bilayer formation with interferometric scattering microscopy. ACS Nano 7, 10662-10670 (2013).

27. Bellve, K., Standley, C., Lifshitz, L. \& Fogarty, K. Design and Implementation of 3D Focus Stabilization for Fluorescence Microscopy. Biophys. J. 106, 606a (2014).

28. Janesick, J. R. Photon transfer. (SPIE Press, 2007).

29. McFarlane, N. J. \& Schofield, C. P. Segmentation and tracking of piglets in images. Machine Vision and Applications 8, 187-193 (1995).

30. Larson, J. et al. Design and construction of a multiwavelength, micromirror total internal reflectance fluorescence microscope. Nat. Protoc. 9, 2317-2328 (2014).

31. de Wit, G., Danial, J., Kukura, P., Wallace, M. Dynamics label-free imaging of lipid nanodomains. Proc. Natl. Acad. Sci. U.S.A. 112, 12299-12303 (2015).

32. Redding, B., Choma, M. A. \& Cao, H. Speckle-free laser imaging using random laser illumination. Nat. Photon. 6, 355-359 (2012).

\section{Figure Legends}

Figure 1. Concept and capabilities of interferometric scattering microscopy (iSCAT). (a) Different detection geometries for scattering and fluorescence microscopy. The pure scattering channel is only a schematic. (b) Typical darkfield detection geometry, where the incident light is efficiently separated from scattered light by total internal reflection. (c) Operating principle of interferometric scattering detection (iSCAT). The light reflected at the sample/glass interface is explicitly collected along with any scattered light from the scatterer.

Figure 2. Recent studies that exploit the unique capabilities of iSCAT. (a) Single molecule myosin 5 motion along actin tracked with a $20 \mathrm{~nm}$ label ${ }^{23}$ with simultaneous $\mathrm{nm}$ precision, $\mathrm{kHz}$ frame rate and unlimited observation time. (b) High-speed nanometric localization of immobilized $20 \mathrm{~nm}$ gold labels imaged at $50 \mathrm{kHz}$ and a representative trajectory consisting of $5 \times 10^{4}$ data points of a $20 \mathrm{~nm}$ label diffusing on top of an artificial 
membrane $^{24}$. (c) Label-free optical detection and imaging of a single protein ${ }^{12}$. (d) Direct observation of supported lipid bilayer formation ${ }^{26}$.

Figure 3. Combined iSCAT and single molecule TIRF experimental setup. The red box contains the illumination and detection path required for iSCAT microscopy. SMF: single mode fiber, T: telescope for beam diameter adjustment MX: adjustable mirrors, AOD: acousto-optic deflectors, TL: telecentric lens, PBS: polarizing beam splitter, QWP: quarter wave plate, OCM: objective coupling mirror, BFP: back focal plane, F1: longpass filter, L1: imaging lens. The telecentric imaging system mapping the beam deflections generated by the AODs into the back focal plane of the objective is magnified for clarity. The blue box contains the optics items required for TIRF illumination, fluorescence imaging and focus stabilization. DMX: dichroic mirrors, D: pick off mirror, F2: fluorescence filter, CyL: cylindrical lens. The elliptical inset depicts the change in the totally internal reflected beam path upon changing the sample-objective distance. This change is mapped onto a second CMOS camera.

Figure 4. Simultaneous single particle tracking with iSCAT and TIRF. (a) Cartoon of a double-labeled myosin 5 assay with a $20 \mathrm{~nm}$ gold particle (red) and quantum dot (blue) each attached to a head domain. (b) Flat-fieldcorrected, background-corrected and corresponding fluorescence image obtained during simultaneous iSCAT and TIRF measurements. Scale bars: $1 \mu \mathrm{m}$. (c) Representative trajectories obtained from iSCAT (red) and fluorescence (blue) channels. Scale bar: $100 \mathrm{~nm}$. (Figure (a) and (c) have been adapted from ref. 23)

Figure 5. Removal of static imaging background. (a) Raw, median and median-divided iSCAT images. The raw iSCAT image displays an inhomogeneous baseline due to uneven illumination and other background sources. (b) Kymographs of $x$ and $y$ cuts through the image recorded as the sample is translated left to right. Both display stripes, which are indicative of sample-independent background contributions. Scale bars: $1 \mu \mathrm{m}$. (c) Division of the raw (orange) by the median (blue) drastically reduces the image background as illustrated for a horizontal cut shown in (a). (d) Removal of additional large-scale features due to slowly varying fluctuations by pseudo flat-fielding. Scale bars: $5 \mu \mathrm{m}$. (e) Revealing dynamic features much smaller than the imaging background by median filtering. Note the 30 -fold reduction in vertical scale in the final image. (f) Consecutive differential imaging for removal of imaging background. Scale bars: $1 \mu \mathrm{m}$.

Figure 6. Cage assembly for fiber coupling. The aspheric condenser is mounted in a cage system and the fiber adapter plate held in a z-translation mount to enable precise coupling of the beam into the fiber.

Figure 7. Microscope assembly. (a) The objective is mounted by threading a hole in an optical breadboard complementary to that of the objective. (b) The xy stage is then secured to the breadboard. Focusing is performed by a linear translation stage with a piezo element mount ay 90 degrees to the plane of the breadboard. The purpose of the mirror mount is to correct for any tilt introduced into the sample plane. It is important to note that are a wide range of commercially available nanopositioning piezo stages that could be used for fine focus control.

Figure 8. AOD placement. The first AOD is mounted on a post and post holder to adjust the height of the crystal element with respect to the beam. The second AOD is then mounted perpendicular to the first on a rotation platform so that the angle of the crystal element may be adjusted.

Figure 9. Concept of beam scanning pulse sequence and illumination. (a) Triggering sequence for AOD scanning. Both AODs rapidly scan the beam across the sample at slightly different frequencies during the camera exposure time $t_{\text {exp. }}$. At the end of the camera exposure the function generators are reset to scan across the sample during the next exposure. (b) Resulting scan pattern across the sample during a single exposure.

Figure 10. Anticipated shot noise-limited behavior for sample system. (a) Flat-field corrected iSCAT image of 20 $\mathrm{nm}$ gold nanoparticles immobilized on a glass coverslip in water. Small variations in the background signal that move together with sample displacements correspond to imperfections of the cover glass substrate. Scale bar: $1 \mu \mathrm{m}$. (b) The top trace shows the fitted position of both gold nanoparticles as a function of time. The modulation in both traces is caused by vibrations extrinsic to the sample. The bottom trace shows the fluctuation in the distance between the two nanoparticles as a function of time. (c) Localization precision as a function of illumination intensity for $20 \mathrm{~nm}$ gold nanoparticles. An increase in illumination intensity allows higher localization precision for a given exposure time. The line shows the expected behavior for a shot noise limited process. 


\section{Supplementary Information Legends}

Supplementary Video 1. Defocusing beam profile for an aligned microscope at step 27. For an aligned microscope and an unscanned illumination, the beam profile defocuses concentrically without displacements and exhibits high degree of radial symmetry as the sample is brought in and out of focus by displacing the axial translation stage.

Supplementary Video 2. Defocusing beam profile for a misaligned microscope at step 27. For a misaligned microscope and an unscanned illumination, the beam profile exhibits lateral displacements and poor radial symmetry as the sample is brought in and out of focus by displacing the axial translation stage. 
a

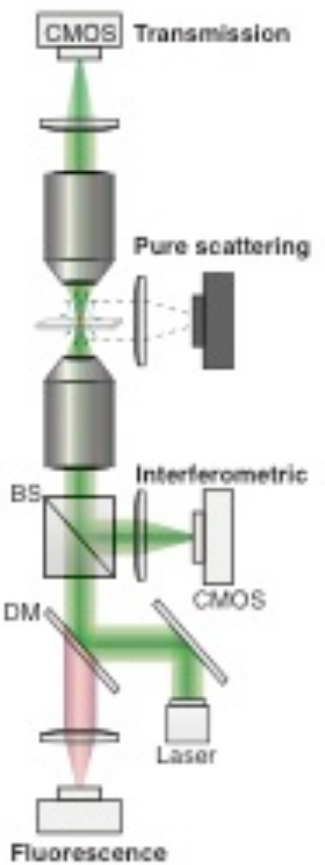

b

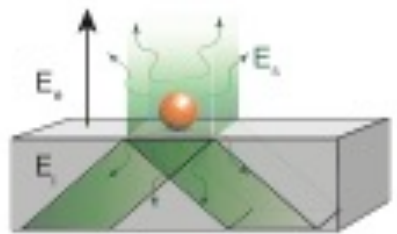

$c$

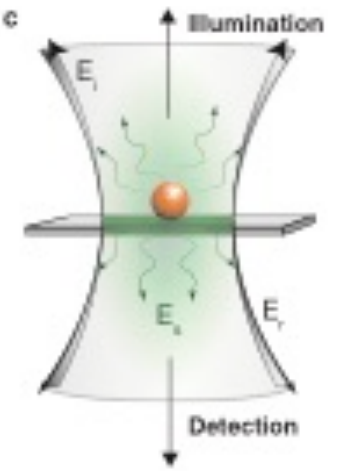

Figure 1 


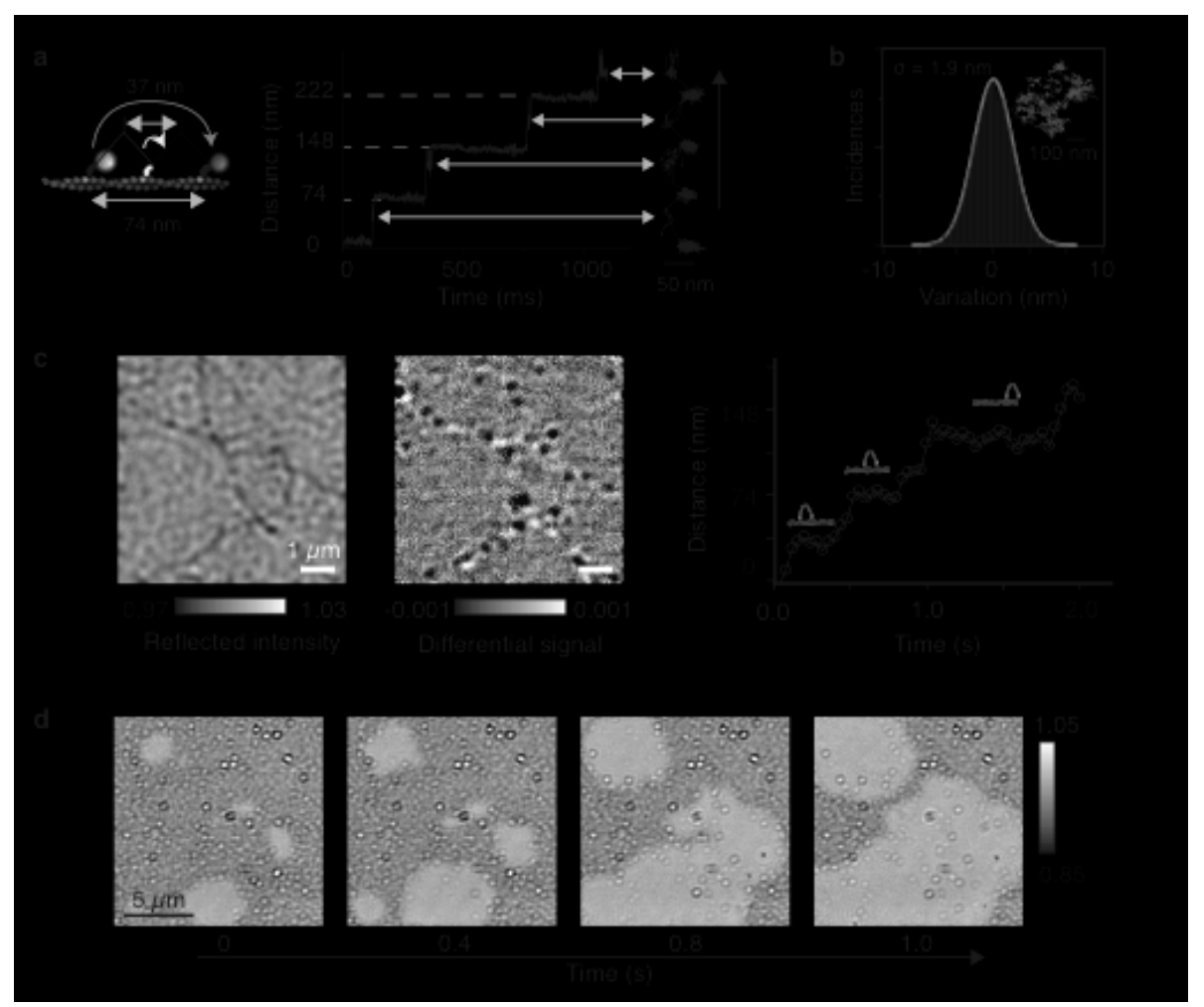

Figure 2 


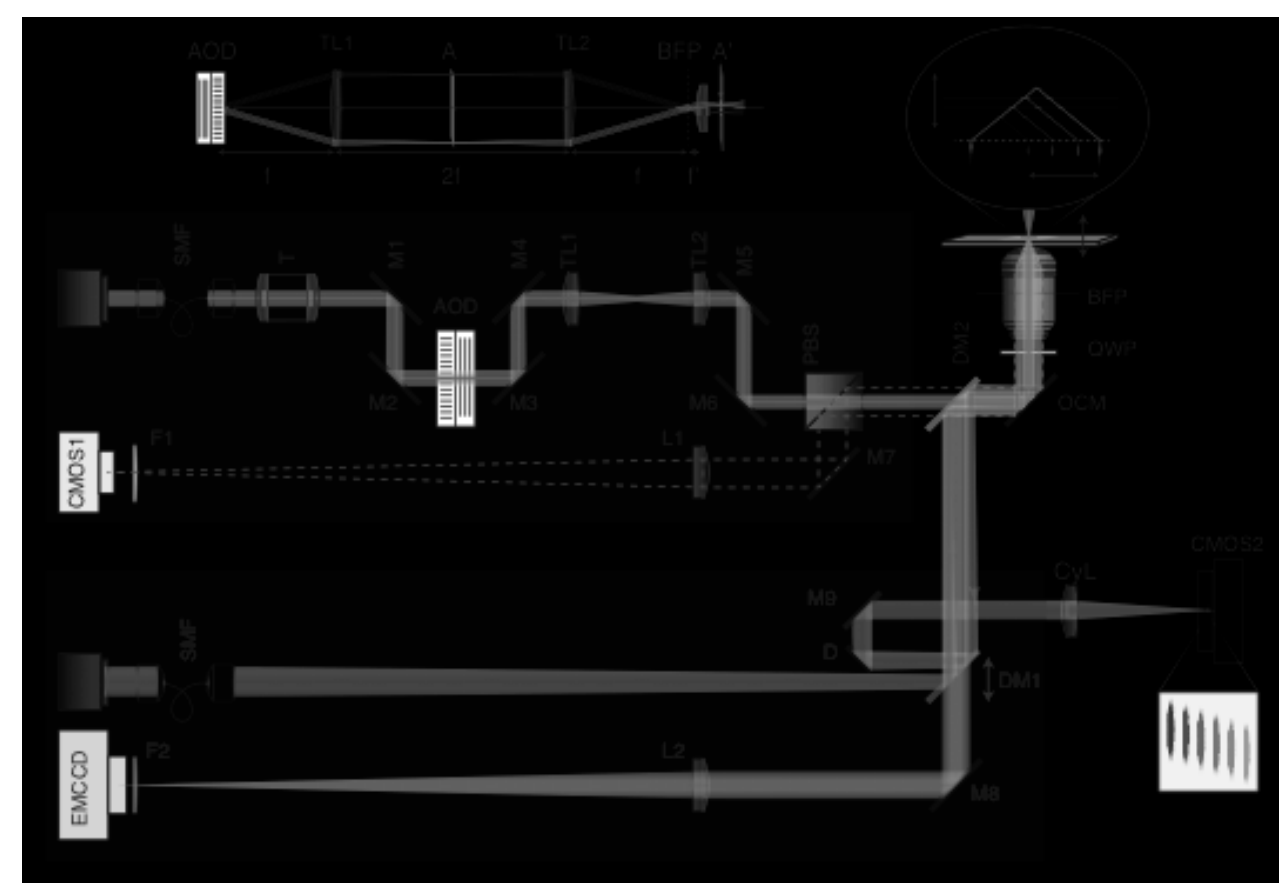

Figure 3 


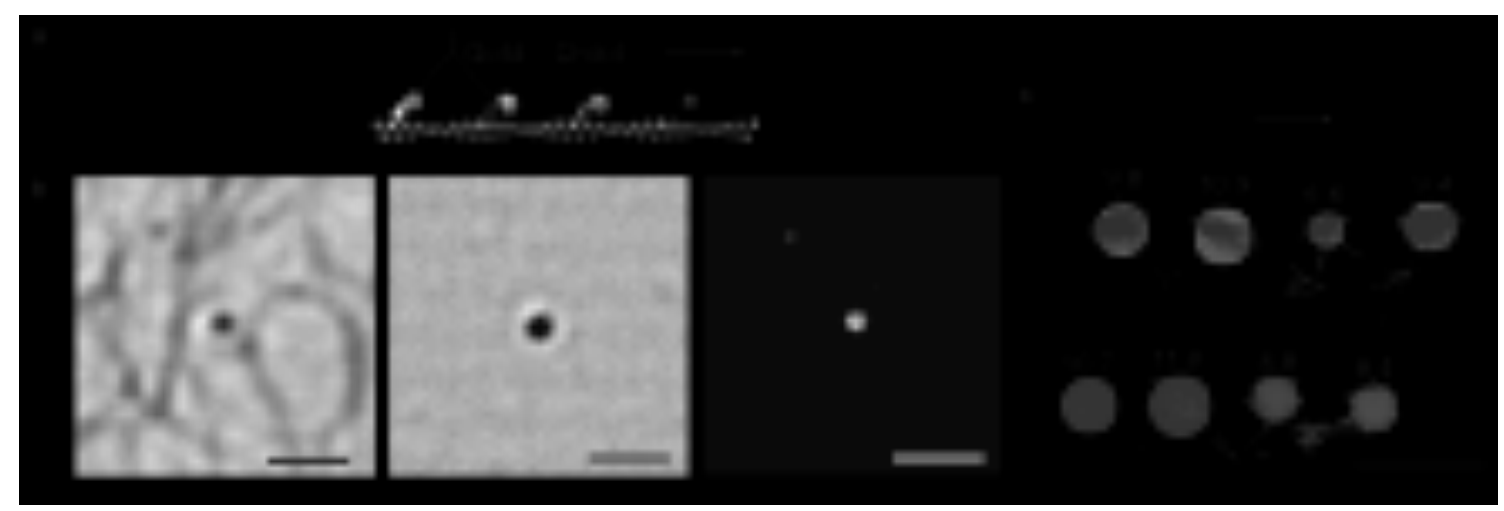

Figure 4 

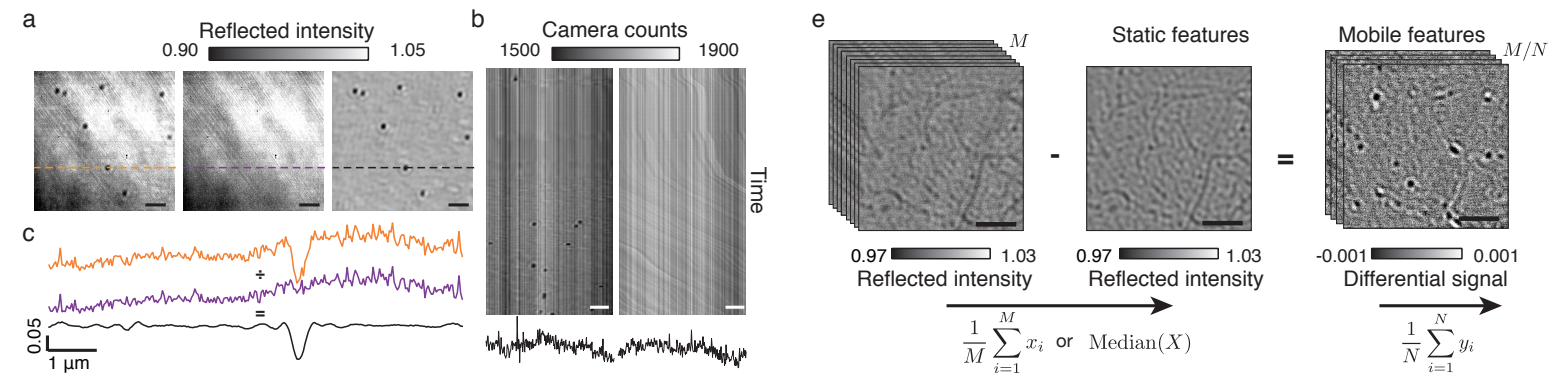

d
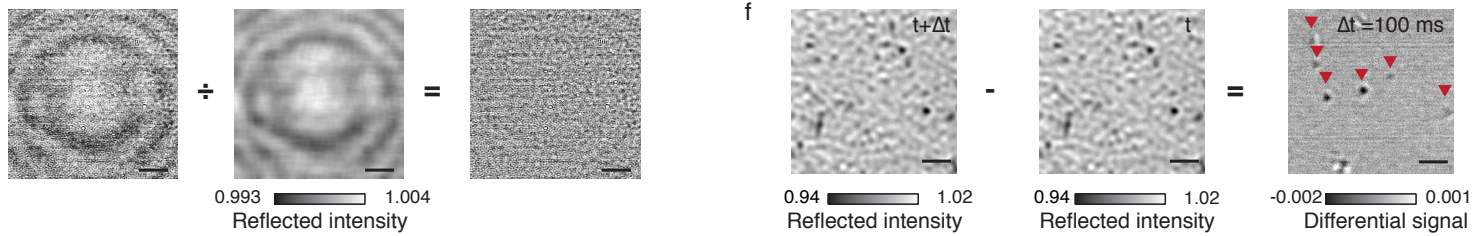

Figure 5 


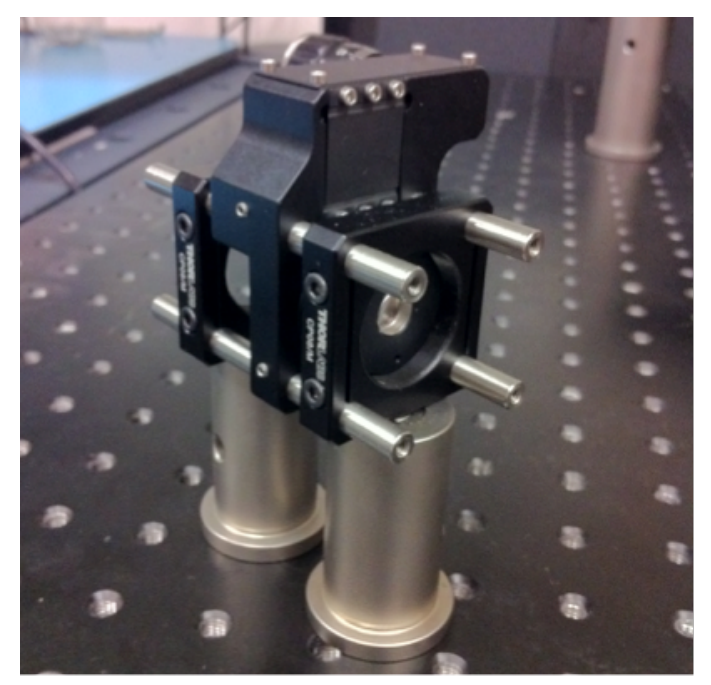

Figure 6 


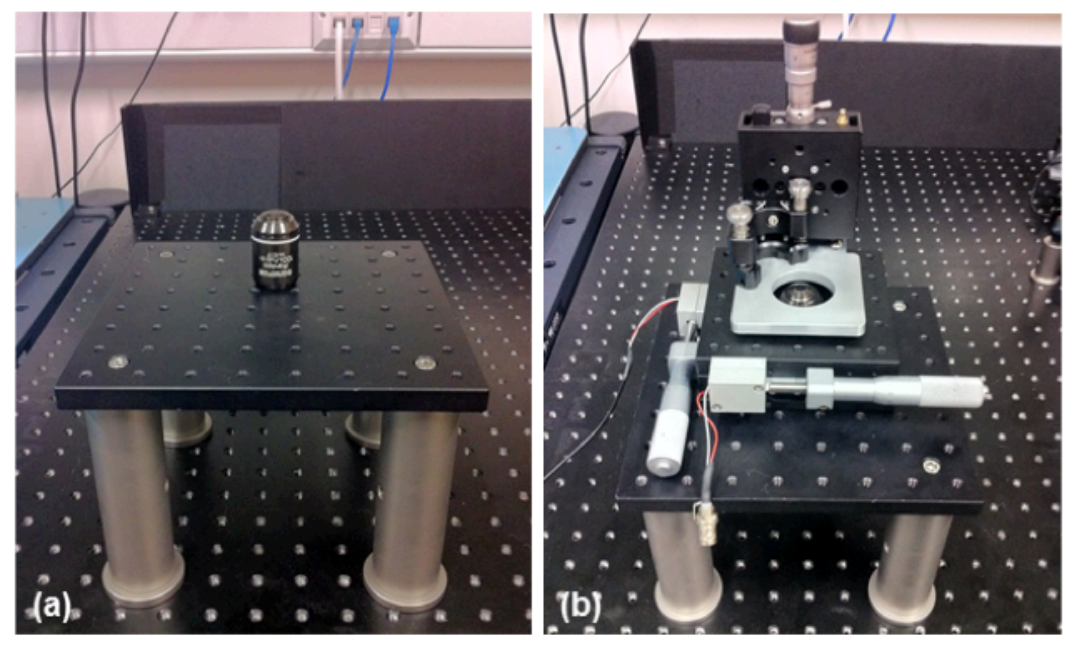

Figure 7 


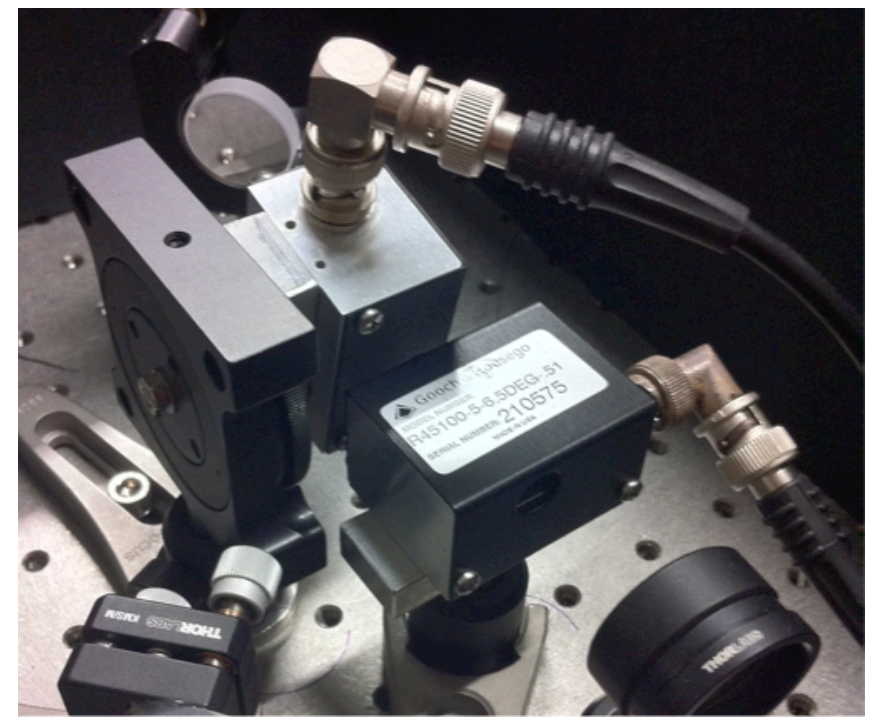

Figure 8 


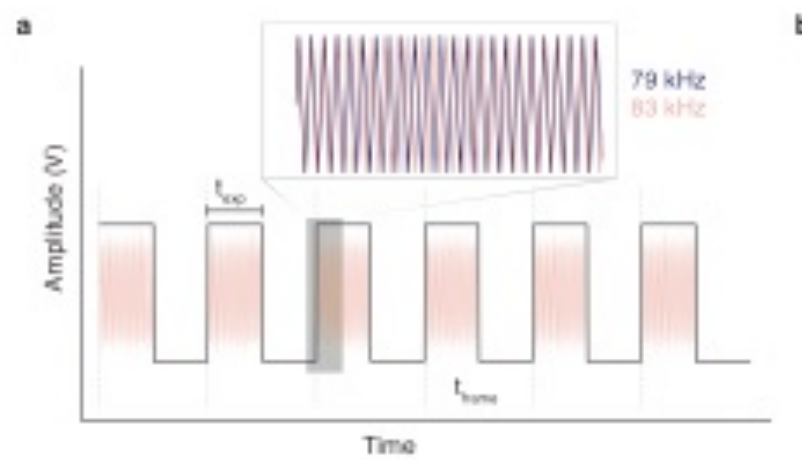

b

Figure 9 


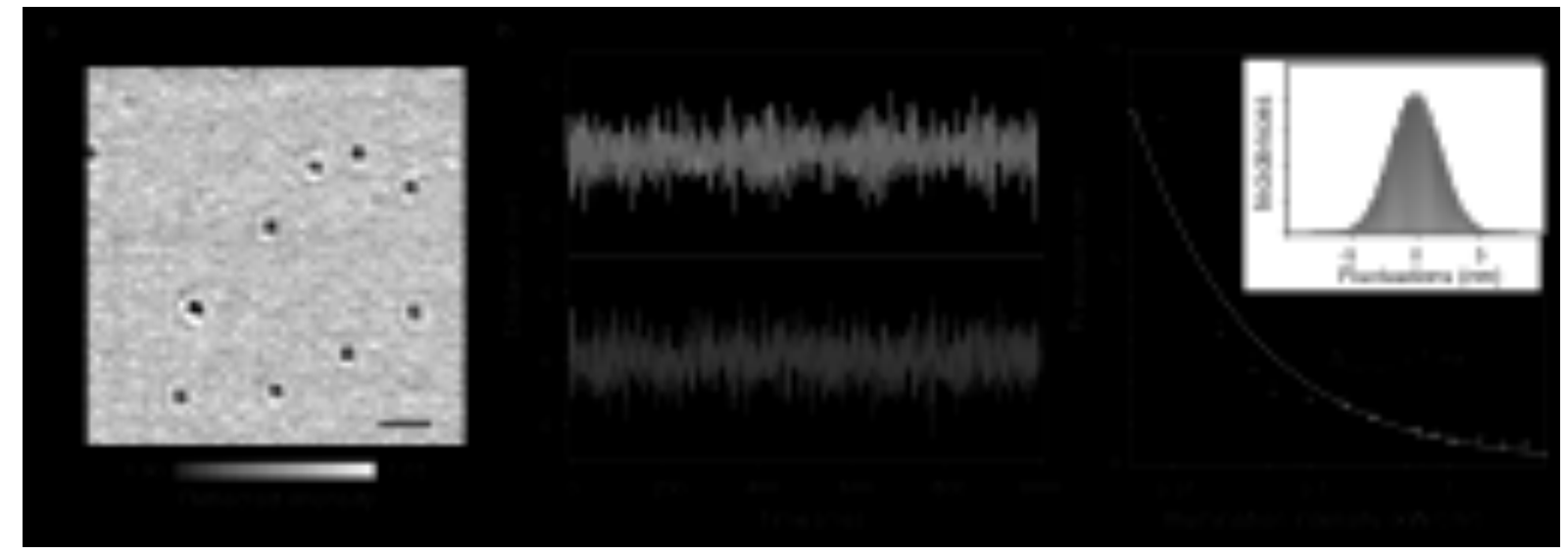

Figure 10 